\section{Sami Haddadin Alin Albu-Schäffer Gerd Hirzinger}

Institute of Robotics and Mechatronics,

DLR-German Aerospace Center,

P.O. Box 1116, D-82230 Wessling,

Germany

\{sami.haddadin, alin.albu-schaeffer, gerd.hirzinger\}@dlr.de

\begin{abstract}
Physical human-robot interaction and cooperation has become a topic of increasing importance and of major focus in robotics research. An essential requirement of a robot designed for high mobility and direct interaction with human users or uncertain environments is that it must in no case pose a threat to the human. Until recently, quite a few attempts were made to investigate real-world threats via collision tests and use the outcome to considerably improve safety during physical human-robot interaction. In this paper, we give an overview of our systematic evaluation of safety in human-robot interaction, covering various aspects of the most significant injury mechanisms. In order to quantify the potential injury risk emanating from such a manipulator, impact tests with the DLR-Lightweight Robot III were carried out using standard automobile crash test facilities at the German Automobile Club (ADAC). Based on these tests, several industrial robots of different weight have been evaluated and the influence of the robot mass and velocity have been investigated. The evaluated non-constrained impacts would only partially capture the nature of human-robot safety. A possibly constrained environment and its effect on the resulting human injuries are discussed and evaluated from different perspectives. As well as such impact tests and simulations, we have analyzed the problem of the quasi-static constrained impact, which could pose a serious threat to the human even for low-inertia robots under certain circumstances. Finally, possible injuries relevant in robotics are summarized and systematically classified.
\end{abstract}

KEY WORDS—Physical Human-Robot Interaction, Simulation, Flexible Arms, Mechanics, Design and Control, Force Control

The International Journal of Robotics Research Vol. 00, No. 00, Xxxxxxxx 2009, pp. 000-000 DOI: $10.1177 / 0278364909343970$

(c) The Author(s), 2009. Reprints and permissions: http://www.sagepub.co.uk/journalsPermissions.nav

Figures 1-10, 12-18 appear in color online: http://ijr.sagepub.com

\title{
Requirements for Safe Robots: Measurements, Analysis and New Insights
}

\section{Introduction}

Bringing robots and humans spatially together as exemplified in Figure 1 leads to the fundamental concern of how to ensure safety for the human. Asimov (1954) noted over 50 years ago that safety should have priority when robots interact with humans. The assurance of safety involves various aspects ranging from preventing electrical threats to coping with human errors. In this paper, however, we focus on various aspects of physical human-robot contact and its related injury potential. Figure 2 gives an initial overview of the relevant contact scenarios which could potentially lead to human injury. Here, we differentiate between unconstrained impacts, clamping in the robot structure, constrained impacts, partially constrained impacts, and resulting secondary impacts. During each of the depicted collisions various injury sources may be present, including fast blunt impacts, dynamic and quasi-static clamping, or cuts by sharp tools ${ }^{1}$. In this paper we address the most relevant injury mechanisms for various contact scenarios and analyze them in detail in order to fully understand the important factors behind them. Intuitively it seems clear that a robot moving at maximal speed (e.g. due to malfunction) can cause severe injury, especially if the impact is unforeseen ${ }^{2}$. On this aspect we present new results, leading to unexpected conclusions.

In this paper we focus on the direct consequences caused by the physical contact between robot and human. Secondary impacts will be left for future research. Since clamping in the robot structure is basically equivalent to constrained impacts and partially constrained impacts, a separate analysis will not be carried out at this point. Furthermore, in most cases evaluating unconstrained and constrained impacts already provides coverage for the worst-case. Thus, we focus on an in-depth evaluation of these major classes in this paper.

1. Please note that in Figure 2 no differentiation between blunt or sharp contact is made since the contact scenario itself remains unchanged in this context. 2. During our evaluation we assume that the impact is unforseen, i.e. the human shows no reaction to reduce the injury potential. 

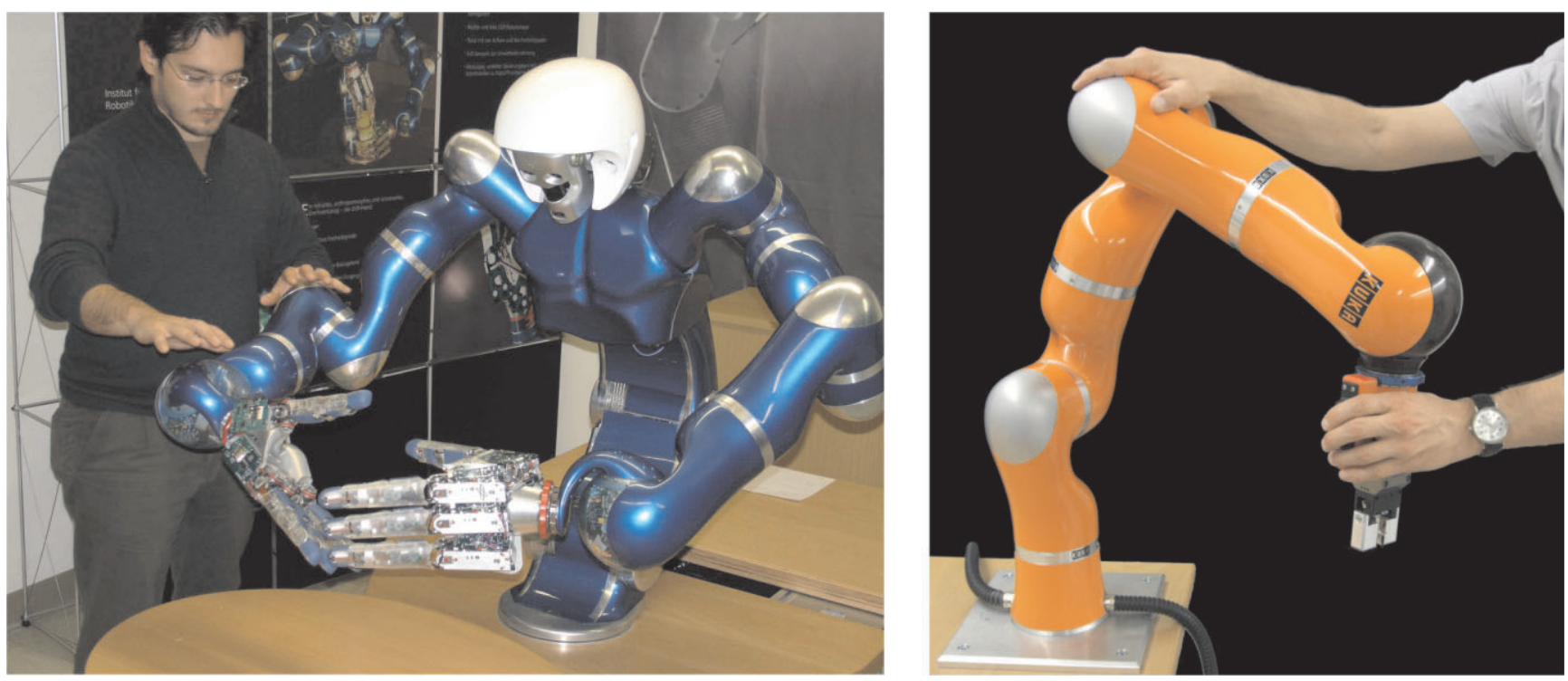

Fig. 1. Physical cooperation between humans and robots is desirable for future robotic applications and poses the fundamental question of how to ensure safety for the human during such scenarios. As an example a human is interacting with the DLR humanoid Justin (left) and the KUKA Lightweight Robot (right). The KUKA Lightweight Robot is based on the DLR-Lightweight Robot III (LWRIII) and is the result of a technology transfer from DLR to the robot manufacturer KUKA.

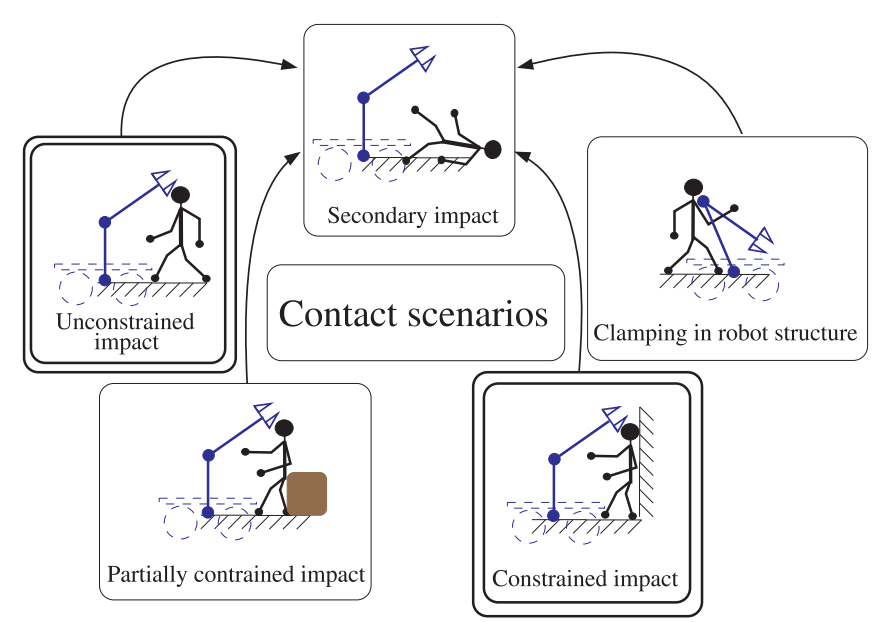

Fig. 2. Classification of undesired contact scenarios between human and robot.

We first give a brief overview on safety in human-robot interaction to lead into our research.

\section{Related Work}

Concerning injuries caused by robots, only very little data and literature are available. United Auto Workers (2004) is a United Auto Workers (UAW) union report which provides raw data on various injuries related to robot operations. It indicates that a majority of injury occurrences involve clamping of a human body part. Further information is presented in SMErobot (2009) which gives data on industrial robot injuries related to the injured body part.

Yamada et al. (1996) evaluated human pain tolerance on the basis of human experiments. In this work somatic pain was considered as a suitable criterion for determining a safety limit against mechanical stimuli. Corke (1999) also discussed robot safety in human environments and pointed out various potential threats.

Pioneering work on human-robot impacts under certain worst-case conditions and resulting injuries was carried out by Bicchi and Tonietti (2004) and Zinn et al. (2004), evaluating free rigid impacts at a robot speed of $1-2 \mathrm{~m} \mathrm{~s}^{-1}$. Both contributions introduced new intrinsically compliant joint design concepts. They also made the first attempt to use the Head Injury Criterion (HIC) (Versace 1971) to quantify the injury potential during occurring collisions. A correction to the initial misinterpretation in units ${ }^{3}$ committed by Bicchi and Tonietti (2004) and Zinn et al. (2004) was first carried out by Haddadin et al. (2007a,b) and then by Bicchi et al. (2008) and Shin et al. (2008).

Haddadin (2005) reviewed various severity indices for the head (Gadd Severity Index (GSI), Maximum Power Index (MPI), Effective Displacement Index (EDI), Revised Brain

3. Instead of using the appropriate units "g" for acceleration, they used $\mathrm{m} \mathrm{s}^{-2}$. 
Model (RBM), Vienna Institute Index (JTI), and Maximum Mean Strain Criterion (MSC)) and for the chest (Acceleration Criterion (AC), Compression Criterion (CC), and Viscous Criterion (VC)) and analyzed these indices in simulation for the case of unconstrained impacts with a lightweight robot. The main conclusion was that during blunt impacts, no significant injury can be observed by means of these criteria at an impact velocity of $1 \mathrm{~m} \mathrm{~s}^{-1}$ with the DLR-Lightweight Robot III (LWRIII). Furthermore, it is shown that a reduction in joint stiffness for an already moderately flexible robot such as the LWRIII (and similar reflected inertia), only marginally reduced the impact dynamics during a rigid impact, e.g. between a robot and the human head.

ISO10218 was introduced to define new collaborative operation requirements for industrial robots (ISO10218 2006). It states that one of the following conditions always has to be fulfilled to allow human-robot interaction: the Tool Center Point (TCP)/flange velocity must be at most $0.25 \mathrm{~m} \mathrm{~s}^{-1}$, the maximum dynamic power at most $80 \mathrm{~W}$, or the maximum static force at most $150 \mathrm{~N}$. Our results demonstrated that these requirements tend to be unnecessarily restrictive and overly undifferentiated, and therefore strongly limit the performance of the robot. We provide a better differentiated analysis in this paper, pointing out the relevant factors, which should be evaluated to give fundamental insight.

Further aspects concerning safety in human-robot interaction were introduced by Ikuta et al. (2003). In this work several danger indices were proposed based on the design properties of the robot. Heinzmann and Zelinsky (2003) proposed a control scheme to limit the impact force of a robot by restricting the torque commands. Lim and Tanie (2000) developed various design aspects for a mobile robot by introducing physical compliance in its trunk and a passively movable base. Kulic and Croft (2007) developed an integrated human-robot interaction strategy incorporating a definition of danger by means of reflected inertia, relative velocity and the distance between human and robot. Recently, Oberer and Schraft (2007) utilized Finite Element Method (FEM) simulations for an evaluation of robot-dummy impacts with an industrial robot.

Attempts to investigate real-world threats via impact tests at standardized crash test facilities in order to analyze safety issues during physical human-robot interaction were to the best of the authors' knowledge first carried out by Haddadin et al. (2007b). In order to quantify the potential danger emanating from the LWRIII, impact tests at the Crash Test Center of the German Automobile Club (ADAC) were conducted and evaluated. The effect of robot speed, robot mass, and constraints in the general environment on safety in human-robot impacts are analyzed in Haddadin et al. (2008b) and Haddadin et al. (2008c).

\section{Outline}

The outcome of the dummy crash tests of Haddadin et al. (2007b) indicated a very low injury risk with respect to the evaluated injury criteria for rigid impacts with the LWRIII. This is confirmed by blunt impact experiments with a human volunteer. Further analysis shows that a robot, even with arbitrary mass moving up to approximately $2 \mathrm{~m} \mathrm{~s}^{-1}$ does not pose a threat to a non-clamped human head with respect to typical head severity indices ${ }^{4}$. First results indicating these implications were given by Haddadin (2005). In this paper we confirm these strong statements by crash tests with several industrial robots. These experiments lead us to other injuries which appear more relevant in the case of unconstrained impacts. After evaluating such free impacts between humans and robots, we analyze dynamic clamping. This is a major source of serious injuries, especially for massive robots. Apart from such dynamic clamping impacts we identify certain situations in which low-inertia robots such as the LWRIII can become seriously dangerous. These situations are related to clamping close to singularities where the robot is able to exert very large forces on the environment as well as on the human.

Finally, an overview of possible injuries, a classification attempt, and related severity measures will be outlined with the goal of assembling a complete view of injury mechanisms in robotics which is missing in the literature up to now.

The influence of joint stiffness in the context of safety in human-robot interaction is a major issue and received significant attention (Bicchi and Tonietti 2004; Zinn et al. 2004). We give some new results concerning the role of joint stiffness during rigid blunt impacts, e.g., with the human head.

This paper is organized as follows. In Section 4 a very short review on injury quantification and classification in automobile crash testing is given. In Section 5 the results of the standardized crash tests at the ADAC are described. These tests motivated a more general analysis consisting of impact tests with industrial robots, and a clamping analysis for blunt dynamic impacts in Sections 6 and 7. In Section 8 clamping in near-singular configurations is addressed and analyzed in more detail. Finally, a categorization of injuries in robotics is given in Section 9.

\section{Quantification and Classification of Injury in Automobile Crash testing}

A large variety of injuries are possible during an accident involving a human and a robot, cf. Figure 3(a). In order to evaluate and categorize all of these possible injuries, a common definition of injury severity is needed. Here, an internationally established definition of injury level and its corresponding

4. Severity indices are injury measures used in the automobile industry. Head injury assessing criteria mostly focus on the evaluation of head acceleration. 


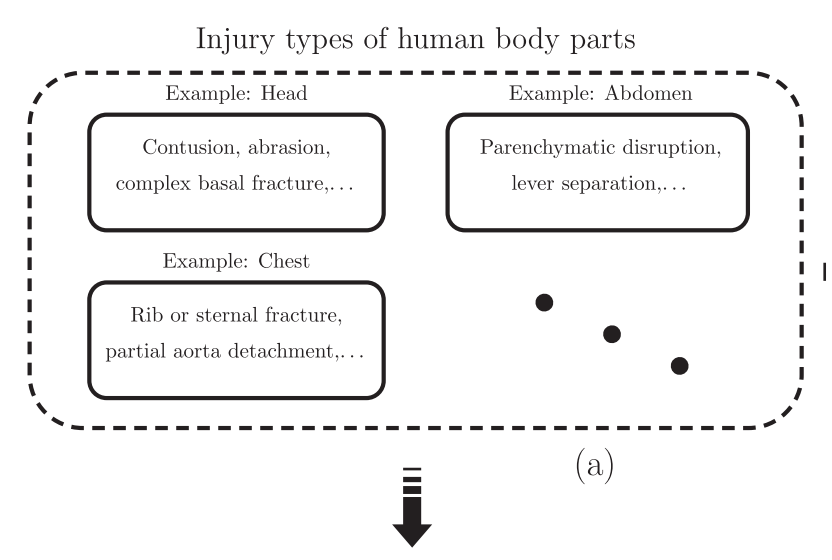

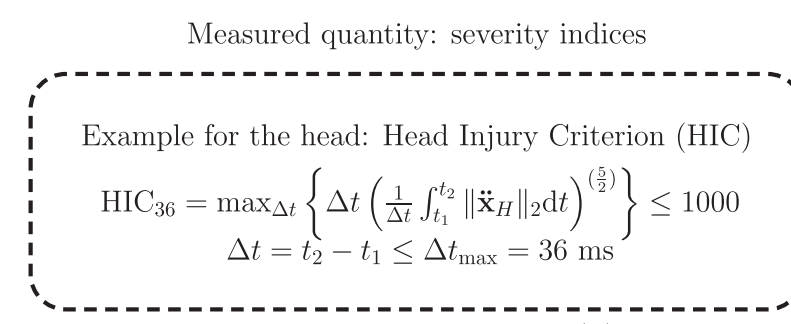

(b)

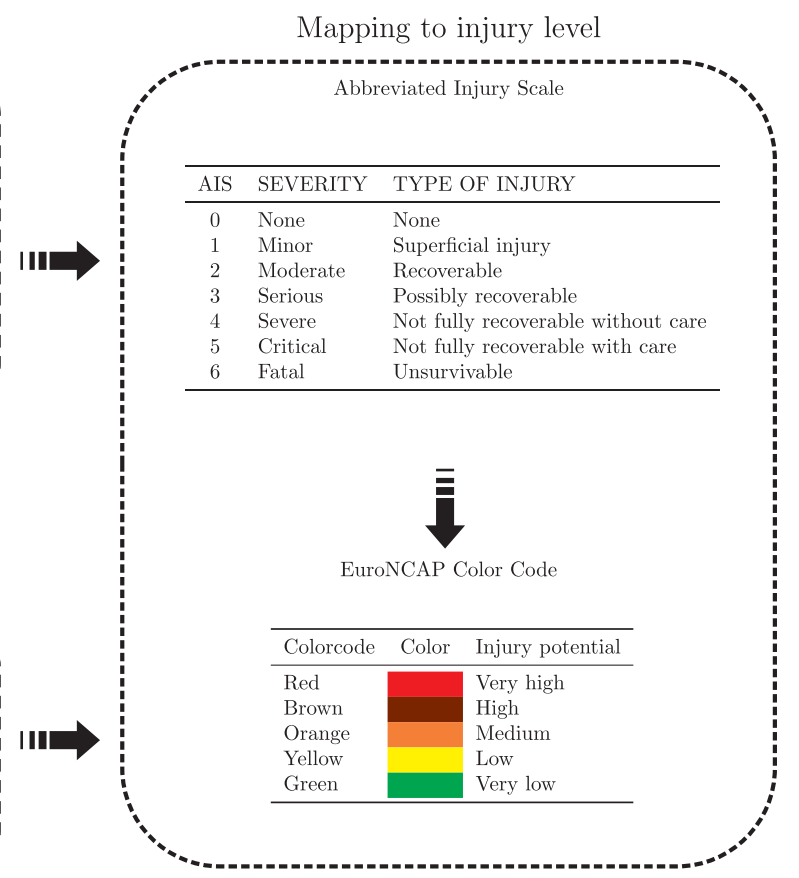

(c)

Fig. 3. The relationship between possible injuries of different body parts, its quantification and classification. Injury types of the human body parts and their severity can be quantified by so-called severity indices. These in turn are mapped to a generic injury level such as the AIS.

pendant in automobile crash testing is used. The Abbreviated Injury Scale (AIS), which is defined in AAAM $(1980,1998)$, subdivides the observed level of injury into seven categories from none to fatal, cf. Figure 3(c).

The European New Car Assessment Programme (EuroNCAP) which is based on the AIS and the American NCAP is the European automobile standard in automobile crash testing. A standardized color code indicates the corresponding injury potential, cf. Figure 3(c) (bottom right).

In order to quantitatively evaluate injury, severity indices are used, which are widely adopted and accepted measures of injury. Each of them is particularly defined for a certain body region. Defining and validating appropriate injury indices for a certain type of interaction is difficult, since it requires the acquisition, biomechanical analysis, and abstraction of data from real human injuries. The biomechanical literature contains a large variety of such indices. However, selecting the appropriate indices for robotics is a challenging task, requiring interdisciplinary knowledge. In the present work we analyze the HIC (Versace 1971) and other indices in order to assess their use and relevance to robotics. Mappings from a severity index to injury level or probability of injury level exist and are usually expressed in AIS/EuroNCAP ${ }^{5}$ levels.

5. For further information on EuroNCAP, HIC, AIS and for the definition of other severity indices (not only for the head but also for the neck and chest), which we evaluated in our work, please refer to Haddadin et al. (2007b).
In the following section, we discuss the important class of blunt unconstrained impacts. First, this is discussed exhaustively by using a robot that was especially designed for physical human-robot interaction: the LWRIII. Apart from discussing various aspects especially relevant for this particular robot general findings are also reported. Furthermore, some comments on the effect that joint stiffness has on safety in pHRI are given.

\section{Blunt Unconstrained Impacts with the LWRIII}

In the following section, the experimental setup at the ADAC, consisting of a LWRIII and a standard frontal Hybrid III crash test dummy (HIII), is briefly described.

\subsection{The LWRIII}

In our evaluation we conducted simulations and experiments with the LWRIII, cf. Figure 1 (right). The LWRIII is a lightweight robot with $1.1 \mathrm{~m}$ reach, moderately flexible joints due to the use of harmonic drives and joint torque sensors, and was explicitly developed for the direct physical interaction and cooperation with humans. The seven-degree-of-freedom (7DOF) 

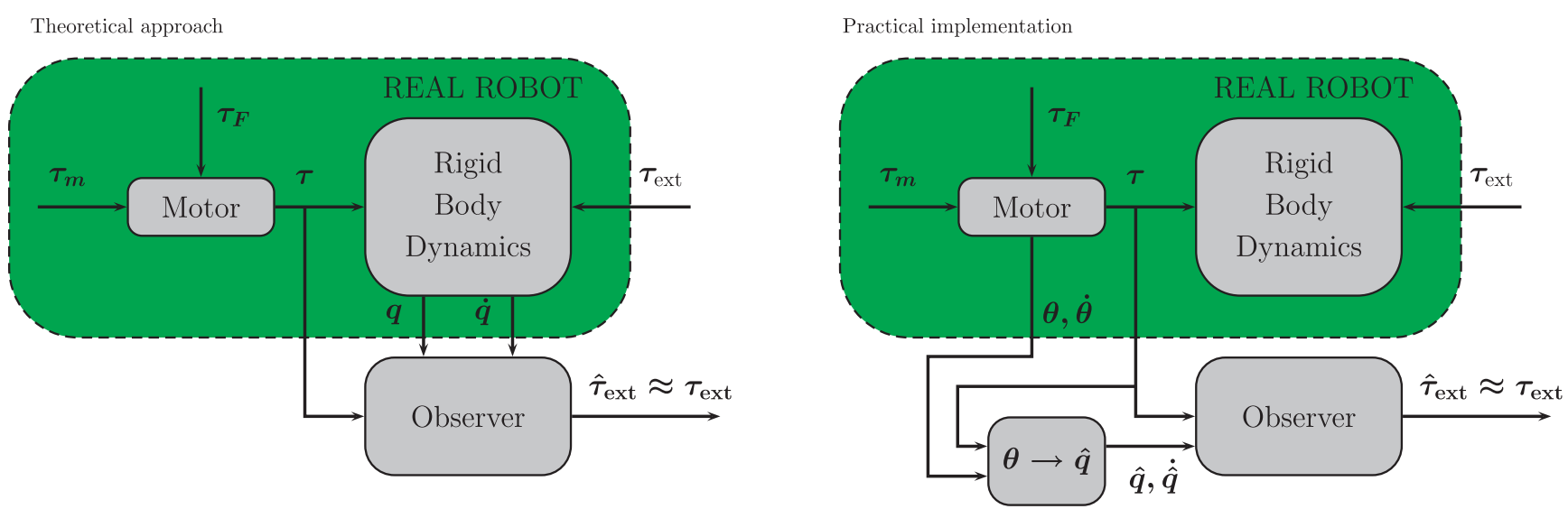

Fig. 4. Structure of the disturbance observer. Here $\boldsymbol{\tau}_{\mathrm{m}}, \boldsymbol{\tau}_{\mathrm{F}}, \boldsymbol{\tau}$, and $\boldsymbol{\tau}_{\mathrm{ext}} \in \Re^{n}$ are the motor torque, friction torque, joint torque, and the external torque, respectively. The disturbance observer inputs are the joint torque, link position, and link velocity. It can be shown that $\hat{\boldsymbol{\tau}}_{\mathrm{ext}} \approx \boldsymbol{\tau}_{\mathrm{ext}}$, meaning for this observer that the estimated external torque, which is the output of the disturbance observer, is a first-order filtered version of the real external torque (left). Since the link side position sensing of the robot is less accurate than the motor position sensing, and due to the good available flexible-joint model of the robot, we use the motor position and velocity $\boldsymbol{\theta}, \dot{\boldsymbol{\theta}} \in \mathfrak{R}^{n}$ as well as the joint model for estimating the link side position and velocity $\hat{\mathbf{q}}, \dot{\hat{\mathbf{q}}} \in \mathfrak{R}^{n}$ (right).

flexible-joint robot has a weight of $14 \mathrm{~kg}$ and a load-to-weight ratio of about one. It is equipped with motor and link side position as well as torque sensors in each joint. The joint torque sensors enable a direct interaction along the entire robotic structure. Details of the design and control methodologies are described by Albu-Schäffer et al. (2007a) and Hirzinger et al. (2004).

A crucial feature of a robot that is designed for physical human-robot interaction has to be an effective physical collision detection and reaction in order to quickly react to external disturbances. Our collision detection algorithm is briefly introduced next.

\subsection{Collision Detection}

The collision detection used is depicted and briefly described in Figure 4 . The detection itself is realized by a disturbance observer that has the estimated generalized momentum as an internal state. It estimates a first-order filtered version $\hat{\tau}_{\text {ext }} \in \Re^{n}$ of the real external torques $\boldsymbol{\tau}_{\text {ext }} \in \mathfrak{R}^{n}$, which gives a robust and fast signal for collision detection. The disturbance observer takes the joint torque, link position, and link velocity as inputs, and observes the generalized momentum $\mathbf{p}=\mathbf{M}(\mathbf{q}) \dot{\mathbf{q}}$ of the robot. Further details are available in De Luca et al. (2006), Haddadin (2005), and Haddadin et al. (2008a).

\subsection{Experimental Setup}

The impact tests were carried out with a HIII dummy. The setup is depicted in Figure 5 (right). It represents the standard equipment used to measure various front crash injury criteria at a sampling frequency of $20 \mathrm{kHz}$. The signals are filtered according to the standardized specifications given in EuroNCAP (2004). In Figure 5 the impact configuration of the LWRIII for head impacts is shown ${ }^{6}$, which was chosen as a trade-off between high maximal impact velocity and large reflected inertia $(\approx 4 \mathrm{~kg})$. The commanded impact velocity was $\|\dot{\mathbf{x}}\|_{\text {TCP }} \in\{0.2,0.7,1.0,1.5,2.0\} \mathrm{m} \mathrm{s}^{-1}$, ranging almost up to the full Cartesian speed of the robot. For these experiments, the robot is additionally equipped with a $1 \mathrm{~kg}$ impactor to provide the contact geometry. A high-bandwidth force (1DOF) and acceleration sensor (3DOF) are mounted on the impactor for measurements. Figure 5 (right) shows the setup of the HIII and the LWRIII.

\subsection{Evaluation and Discussion}

During the experiments at the ADAC, the standard measurements for automotive crash tests which can be acquired with a HIII for the head, neck, and chest were performed. Injury indices for the head are related to its acceleration; those for the neck to forces and torques; and those for the chest to acceleration and deflection.

All calculations of the severity indices were carried out by the ADAC. These calculations were performed according to the EuroNCAP, and are presented in detail in Haddadin et al. (2007b), where the exact definition of the various indices is also given. The present paper focuses on the main conclusions

6. See also Haddadin et al. (2007a) which can be downloaded from http:// www.robotic.de/Sami.Haddadin. 

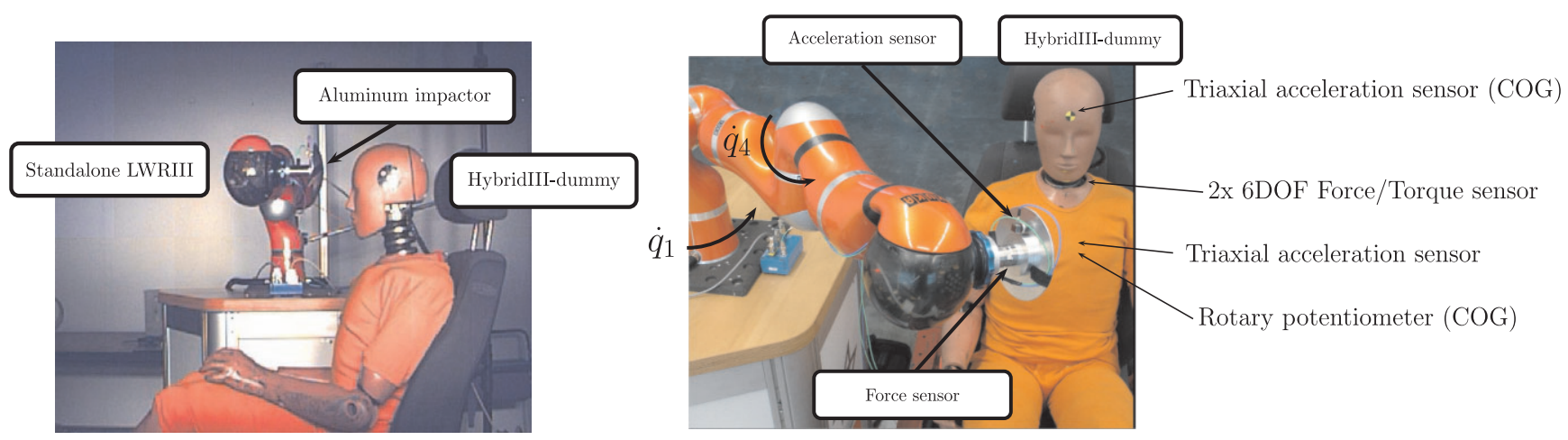

Fig. 5. High-speed recording of the impact tests with a HIII dummy (left). Setup of the LWRIII (with additional external force and acceleration sensor) and the HIII (right).

and the lessons learned from these experiments, which constitute an important piece in the complete puzzle of possible human-robot injuries constructed in this paper.

The main conclusion of the experiments concerning injury severity of humans is that all evaluated severity indices are located in the lowest quarter of the green area in the EuroNCAP color code, as depicted for three particular head, neck, and chest indices in Figure 6 (see also the HIC plot for LWRIII in Figure 13).

This finding can be explained by the fact that the maximal speed of the LWRIII (as with most industrial robots) is considerably lower than typical car velocities. Automotive crash test velocities usually begin at $10 \mathrm{~m} \mathrm{~s}^{-1}\left(\equiv 36 \mathrm{~km} \mathrm{~h}^{-1}\right)$, which is a rather slow car velocity, but is usually not reached by geared robots. Accordingly, the main incidents of injury for car accidents occur at high velocity. Therefore, all indices are tailored to reflect this aspect. More specifically, our evaluation of severity indices such as the HIC clearly indicates that severe injuries can be excluded during free impacts with the robot moving at speeds up to $2 \mathrm{~m} \mathrm{~s}^{-1}$. The correlation to injury probability of the HIC according to NHTSA (1997) indicates that the probability of suffering from less than or equal to minor $^{7}$ injury is $p($ AIS $\leq 1)=7.5 \times 10^{-5} \%$ for the LWRIII at such velocities. This points out that the range of injuries during unconstrained blunt impacts are of very low severity. On the other hand, the need for indicators clearly tailored to low severity injuries seems apparent. To simply use the mapping of HIC to injury probability (NHTSA 1997) for low severity collisions seems not sufficiently differentiated as this criterion was clearly developed for much higher injury levels and intended primarily for separating life-threatening from non-lifethreatening injuries.

Owing to this new focus on minor injuries during free impacts with robots $^{8}$, injury mechanisms must be analyzed that

7. According to the AIS.

8. From now on we implicitly assume that impacts are no faster than $2 \mathrm{~m} \mathrm{~s}^{-1}$ if not stated otherwise. appropriately represent this class of severity. Corresponding indices should also be proposed. This is presented in Section 6.2.

We also draw some further conclusions related to the nature of robot impacts with rigid human body parts such as the head. They give some new insights into questions posed in the robotics literature. An increase in intrinsic safety has been unambiguously related to an introduction of joint compliance in the robotics literature as described by Bicchi and Tonietti (2004) and Zinn et al. (2004). It has been stated that a drastic joint stiffness reduction is desirable to realize a decoupling of the motor from the link inertia. In turn this reduces the reflected inertia during human-robot impacts.

However, it is unclear to what extent joint compliance achieves decoupling, as it is heavily influenced by the contact properties of the human. In this regard our experiments aim to gain some insight into this question. They show that a pure structural compliance (in this case mainly by the harmonic drive and the joint torque sensors) such as that of the LWRIII is already sufficient to realize this desired behavior for typical robot joint inertias.

\subsubsection{Typical Impact Characteristics}

Figure 7 (top) shows the recordings of an impact with the dummy head at $2 \mathrm{~m} \mathrm{~s}^{-1}$. It displays the torque in one joint $\left(\tau_{4}\right)$, as well as the acceleration $\left\|\ddot{\mathbf{x}}_{\mathrm{Al}}\right\|$ and force $\mathbf{F}_{\mathrm{ext}}$ at the tip. The first aspect to be observed is that the impact peak at the contact between robot and head is very short (only 6-10 ms), while the propagation of the impulse over the robot inertia and the joint elasticity leads to a considerable delay in the joint torque peak. The consequences of these observations are discussed next.

\subsubsection{Joint Stiffness and Collision Detection}

Before the joint torque starts to increase, the relevant force/ acceleration peak period is practically over, cf. Figure 8 (left). 

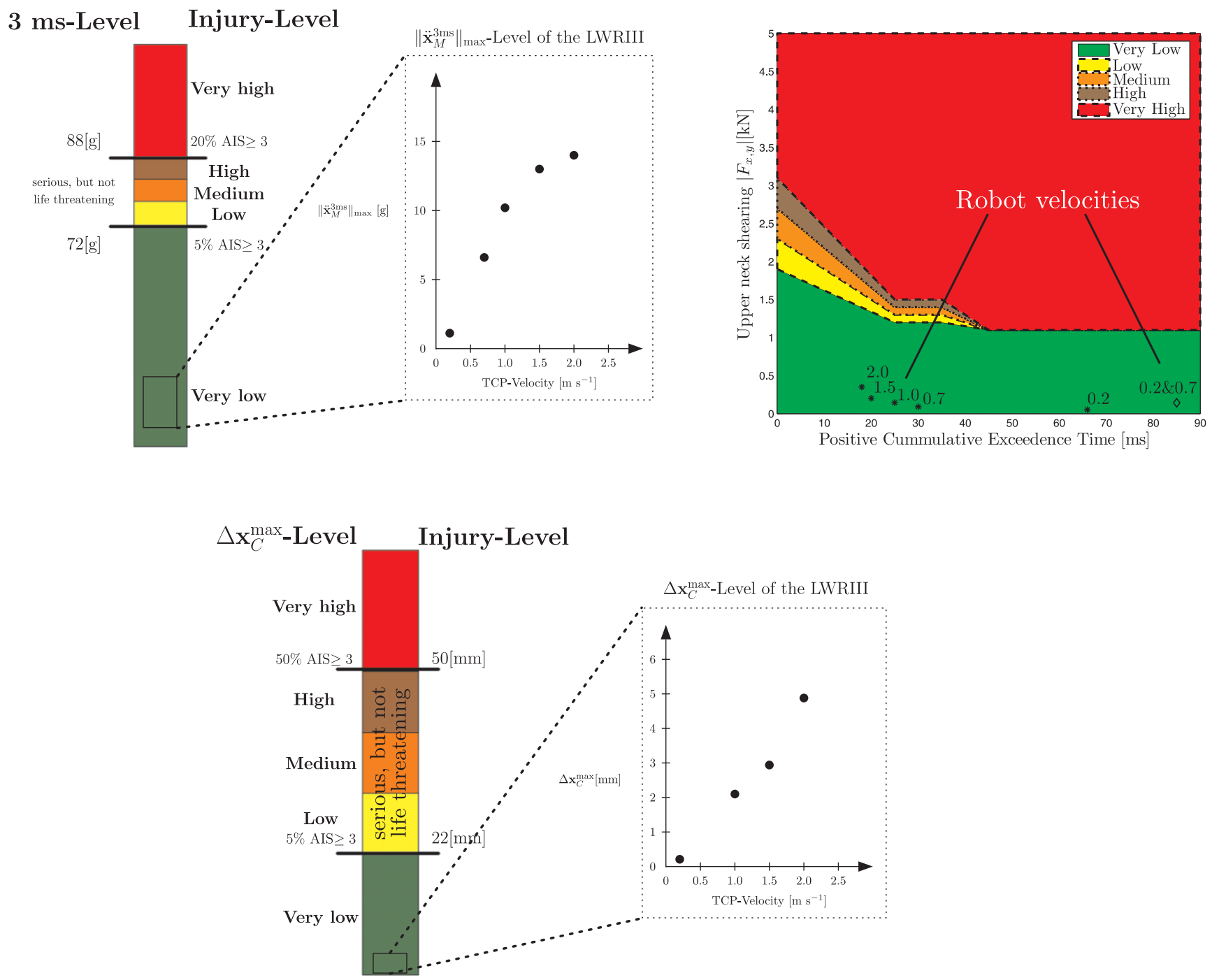

Fig. 6. Resulting $3 \mathrm{~ms}$ criterion for the head (top left), shearing forces $F_{x, y}$ for the neck (top right), and maximum deflection $\Delta \mathbf{x}_{C}^{\max }$ for the chest (bottom). The values were measured for varying impact velocities and rated according to the EuroNCAP Assessment Protocol And Biomechanical Limits.

Thus, during this particular time interval the motor and link inertia are decoupled by the intrinsic joint elasticity, and only the link inertia is involved in the impact. Therefore, decreasing joint stiffness, e.g. via antagonistic actuation, would not have any effect on a (hard contact) head impact with link inertias similar to, or higher than the ones of the LWRIII. In this regard, we are implying that the flexible joint assumption holds for similar lightweight designs ${ }^{9}$. For collisions with softer body parts (e.g. the arm as outlined by Haddadin et al. (2008a)) the impact duration is higher and decreasing joint stiffness might reduce contact forces. To validate this statement, the resulting

9. For a very stiff and heavy industrial robot, for example, this is not the case. contact force was simulated with a dummy head model ${ }^{10}$ and a reduced LWRIII model for three different stiffness values ${ }^{11}$. Our results showed that the contact force (respectively HIC) is practically invariant with respect to a reduction of joint stiffness to values below the one of the LWRIII, cf. Figure 7 (right). The spring force starts increasing well after the maximum contact force was reached when the contact to the head is nearly lost. Therefore, neither the reduction of joint stiffness nor of the motor inertia have an influence on the (very short) impact dynamics even for such joint stiffness as the LWRIII's intrinsic

10. The model is extracted from real impact data.

11. The simulation is one-dimensional, meaning that the reflected motor and link inertia as well as reflected joint stiffness are used to simulate this collision. 

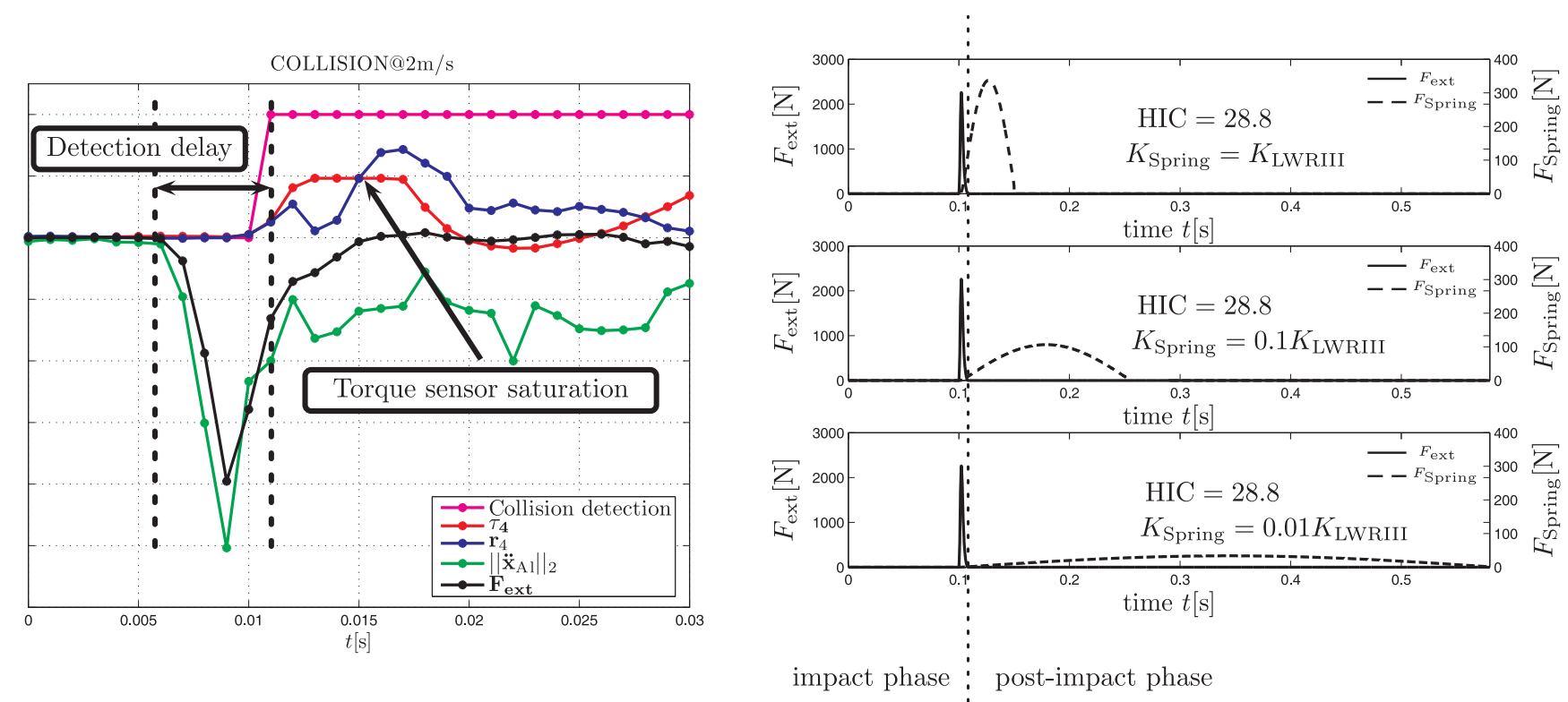

Fig. 7. Impact characteristics at $2 \mathrm{~m} \mathrm{~s}^{-1}$. The plot is intended to show the timing of the signals: while acceleration $\left\|\ddot{\mathbf{x}}_{\text {Al }}\right\|$ and impact force $\mathbf{F}_{\text {ext }}$ are simultaneous, there is a delay in the reaction of the joint torque $\tau_{4}$ and the additional external torque estimation $r_{4}$ to the impact (left). The effect of stiffness reduction on impact force, HIC, and spring force is shown in the lower part. The solid line indicates the contact force and the dashed line the spring force generated by the joint stiffness. The spring force decreases in magnitude and increases in duration when reducing the spring stiffness. The HIC is constant HIC $=28.8$ for all three simulations (right).

stiffness. The link side inertia is the sole dominant influence on the impact force, cf. Figure 8; see also Bicchi and Tonietti (2004).

In order to investigate whether a physical collision detection scheme is able to reduce impact severity, the earlier described collision detection and reaction scheme is used in the experiment and indicated in Figure 7 (left). Alternatively, the acceleration signal of the impactor, i.e. an ideally fast detection, was utilized to trigger several reaction schemes ${ }^{12}$. In both cases the resulting values of the injury indices did not differ from those obtained without any reaction strategy. This is due to the inability of the motors to extract the kinetic energy fast enough to decrease the impact dynamics.

Three main conclusions concerning severity reduction of impact characteristics can be drawn.

1. No physical collision detection and reaction mechanism is fast enough to reduce the impact dynamics of fast and rigid impacts for the considered robot type.

2. For such impacts further joint stiffness reduction does not lower impact forces or severity indices since motor and link inertia are already decoupled.

12. For example, as soon as a collision has been detected the robot switches within one cycle time of $1 \mathrm{~ms}$ from position control to torque control with gravity compensation (Albu-Schäffer et al. 2007b).

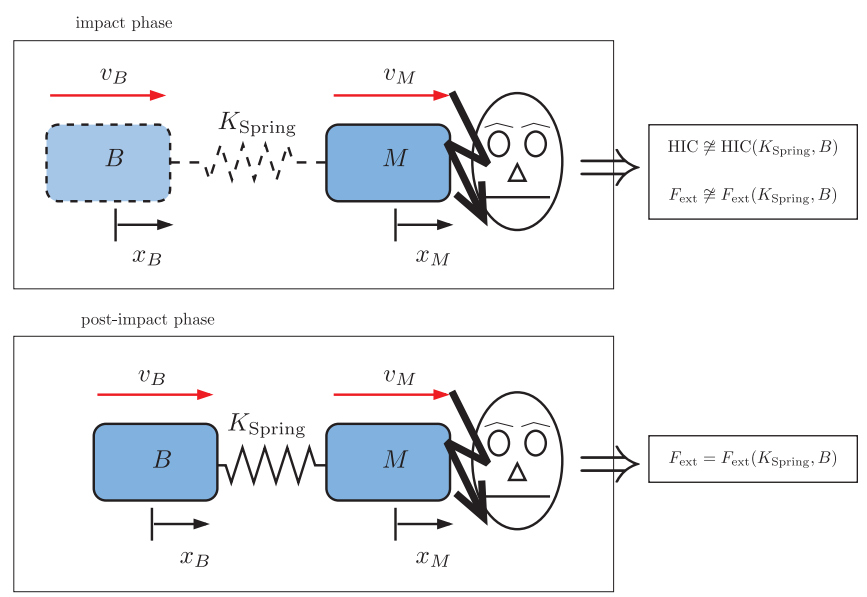

Fig. 8. A rigid impact between a compliant joint and the human head. At moderately high joint stiffness, it is mainly a process between the link inertia and the human head. Note that we refer to the impact phase and post-impact phase in the sense that the former is relevant for the calculation of HIC or maximum impact forces and the latter is not. Refer to Figure 7 (right) for a further comparison. 
3. Soft covering is an adequate countermeasure to reduce the impact effectively.

Apart from these characteristic properties another important observation, seen at an impact velocity of $1 \mathrm{~m} \mathrm{~s}^{-1}$ and above, is that the specified maximum joint torques of the robot were exceeded for several milliseconds during the impact, cf. Figure 7 (left) ${ }^{13}$. This shows that the robot is exposed to enormous loads during such contacts. Countermeasures are needed to ensure the safety of the robot. Speed limitation to subcritical values is one option, others include a reduction in joint stiffness (Haddadin et al. 2007c, 2009) or fast collision reaction strategies. Both measures, which are not effective in protecting the human in case of free impacts, can help to protect the robot joints. This is due to the difference between the duration of the impact itself and the joint torque peak, cf. Figure 7 (right).

Some general remarks can be made concerning the deliberate introduction of mechanical compliance into the robot joints as done in (Morita et al. 1999; Bicchi and Tonietti 2004; Vanderborght et al. 2006).

1. Adding more compliance into the joint does not reduce the impact characteristics significantly for the relatively high intrinsic joint stiffness of the LWRIII.

2. Introducing an elastic joint element makes it possible to store and release energy during motion ${ }^{14}$. By utilizing the intrinsic joint stiffness it is possible to achieve link velocities above motor levels by choosing an appropriate trajectory (Wolf and Hirzinger 2008; Haddadin et al. 2009).

3. Low intrinsic joint compliance can be used to significantly reduce impact joint torques at high impact speeds even with rigid objects and thus protect the robot (Haddadin et al. 2009).

As shown in Section 5.6, impact velocity is the main governing factor during a rigid impact. Thus, a joint design which is intrinsically faster is actually more dangerous by design. It may be said that a compliant joint is more dangerous than a stiff joint in a worst-case scenario (e.g. operated at maximal velocity). Additional control and planning measures have to be taken in order to keep a very compliant joint sufficiently safe in dynamic operation mode.

\subsection{Human-Robot Impacts}

Owing to the encouraging results described in the previous sections and in order to give the ultimate proof for the extremely low injury risk during blunt impacts with the LWRIII,

13. In the robot a mechanical end stop limits the deflection range of the torque sensor which then goes into saturation. A low-level emergency stop is initialized as soon as this event is triggered.

14. Please note, that this is not a discussion about variable joint stiffness but about a low constant joint elasticity in general. impact tests at increasing robot speed were carried out with a volunteer for the chest, abdomen, shoulder, and the head (Haddadin et al. 2007a), see Figure 9. Impact speeds ranged up to $2.7 \mathrm{~m} \mathrm{~s}^{-1}$ for the first three body parts and up to $1.5 \mathrm{~m} \mathrm{~s}^{-1}$ for the head ${ }^{15}$. During the entire experimental series the collision detection was switched off (the detection was activated but the robot was programmed to continue its desired trajectory even in case of a collision). Only a low-level feature of the robot engaged the brakes in the case of exceeding the maximum nominal joint torques of the robot. However, this feature is not able to affect the impact itself due to the delayed increase of the joint torque (see Section 5.4.2).

As predicted by the dummy tests no injury could be observed even at such high speed impacts.

\subsection{Influence of Robot Mass and Velocity}

Since the LWRIII with its lightweight design is specially designed for close cooperation with humans, it is desirable to evaluate the effect of the robot mass on the dynamics of such an impact for a more general class of robots. Apart from the robot's mass, the influence of its velocity is of fundamental interest. Figure 10 shows the dependency of HIC on the robot mass up to $500 \mathrm{~kg}$ with the graphs being parameterized by impact velocities of $\|\dot{\mathbf{x}}\|_{\mathrm{TCP}} \in\{0.2,0.5,1.0,1.5,2.0,2.5,3.0\} \mathrm{m} \mathrm{s}^{-1}$. Two main statements can be deduced:

- HIC saturates with increasing robot mass for each impact velocity;

- impact velocity is the dominant factor in the injury severity.

The first statement was particularly unexpected as it contradicts the intuition of a massive robot being a priori life threatening. An interpretation of the saturation effect can be drawn: whether a very massive robot collides at $2 \mathrm{~m} \mathrm{~s}^{-1}$ with a human head or the human runs at $2 \mathrm{~m} \mathrm{~s}^{-1}$ (which is equivalent to $7.2 \mathrm{~km} \mathrm{~h}^{-1}$ ) against a rigid wall is nearly the same. This very intuitive example shows that one would not be seriously injured, even though this impact occurred at relatively fast walking speed. Therefore, even the infinite mass robot cannot become dangerous at $2 \mathrm{~m} \mathrm{~s}^{-1}$ by means of impact related criteria used in the automobile industry (such as HIC), as long as clamping and impacts with sharp surfaces can be excluded. Let us consider a simple mass-spring-mass model for the impact between human and robot ${ }^{16}$. Here $M_{H}$ and $M_{R}$ are the reflected inertias of the human and robot, $K$ is the contact stiffness which is mainly the stiffness of the human contact area

15. These tests are given as Extensions 1-5 here.

16. For the HIC we assumed a Hunt-Crossley model. However, in order to keep the discussion simple, we assumed a linear spring between the robot and human head mass. 

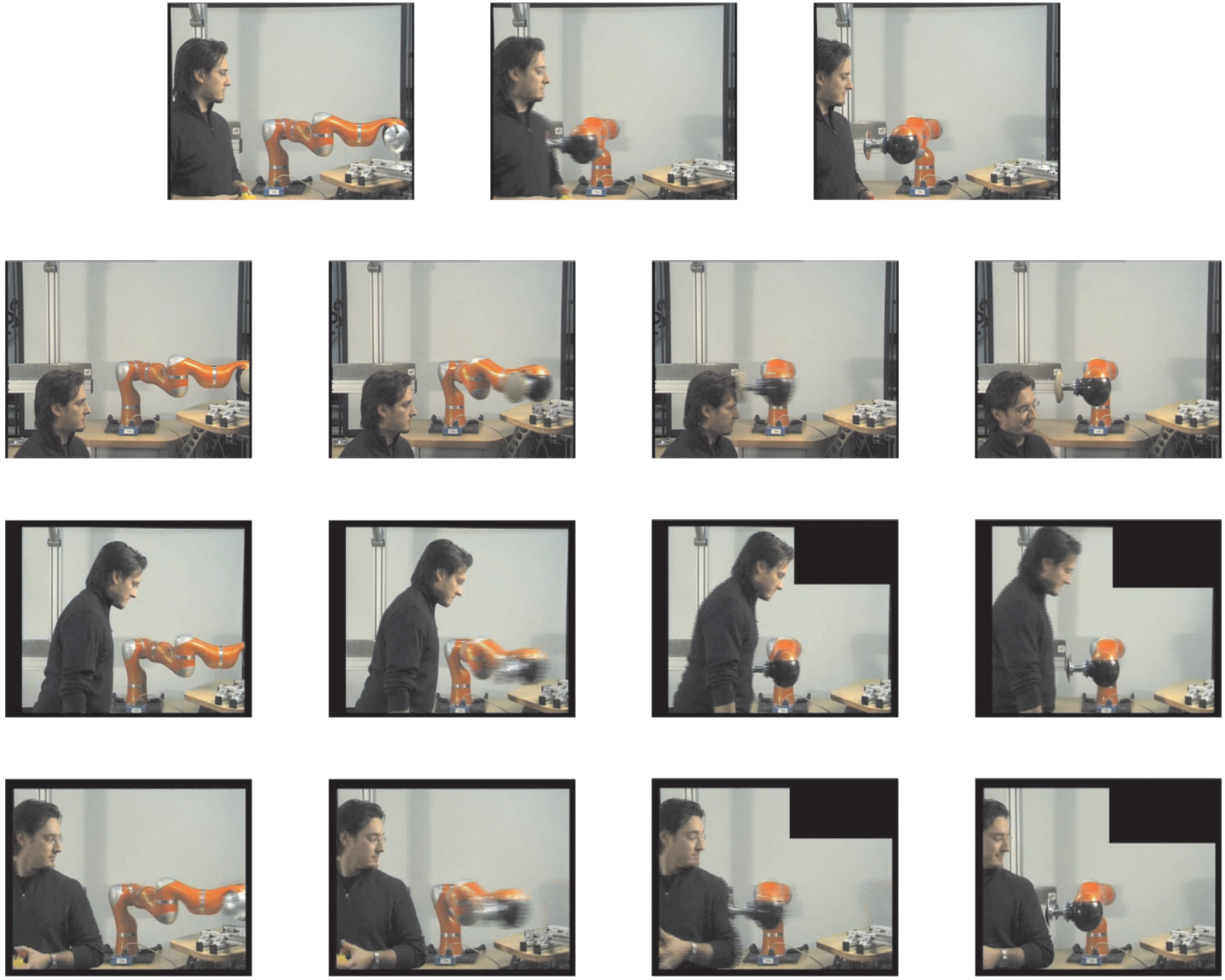

Fig. 9. Impact tests with a human chest at $2.7 \mathrm{~m} \mathrm{~s}^{-1}$ and head at $1.5 \mathrm{~m} \mathrm{~s}^{-1}$. The impact velocities for the abdomen and the shoulder were also $2.7 \mathrm{~m} \mathrm{~s}^{-1}$ which is the maximum velocity of the robot. During all of these experiments the robot does not react to the activated collision detection. The robot stopped only when the maximum nominal joint torques were exceeded. However, as a result of the crash test dummy experiments, the impact forces caused by the very short collision duration cannot be affected by this feature due to its delayed reaction. These tests were initially described in Haddadin et al. (2007a) and support, if not even prove, the conclusions stated previously.

in the case of a rigid robot, $\dot{x}_{R}^{0}$ is the relative impact velocity between the robot and human. Solving the corresponding differential equation leads to the contact force

$$
F_{\mathrm{ext}}= \begin{cases}M_{H} \ddot{x}_{H} & \text { if }|t|<\frac{T}{2} \\ 0 & \text { otherwise }\end{cases}
$$

with

$$
\ddot{x}_{H}=\frac{M_{R}}{M_{R}+M_{H}} \dot{x}_{R}^{0} \omega_{n} \cos \left(\omega_{n} t\right),
$$

where

$$
\omega_{n}=\sqrt{\frac{M_{R}+M_{H}}{M_{R} M_{H}} K}
$$

and

$$
T=\frac{2 \pi}{\omega_{n}} .
$$

The maximum value of this force is consequently 

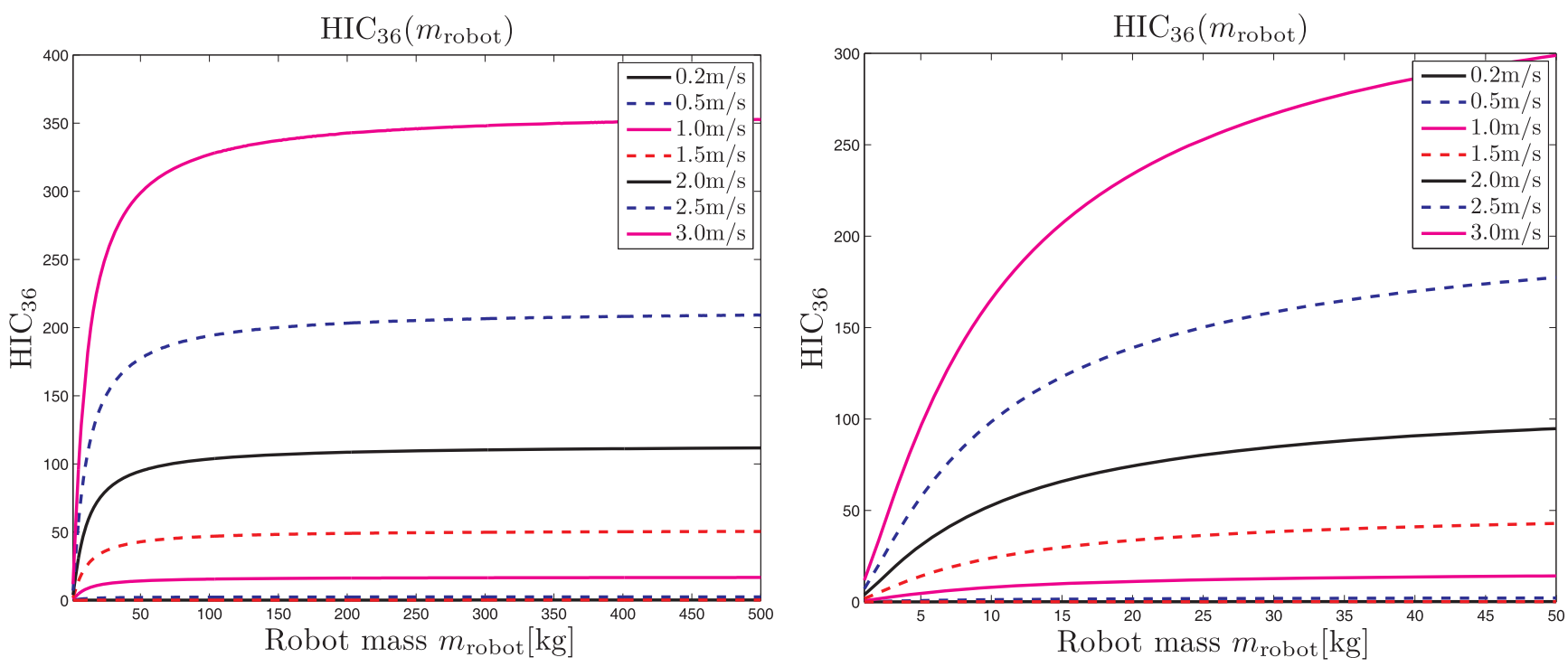

Fig. 10. Resulting HIC calculated from simulated 1DOF impacts between a robot with increasing mass and a dummy head model extracted from real impact data. A saturation effect can be clearly observed with increasing robot mass. Only the impact velocity is relevant above a certain robot mass.

$$
\begin{aligned}
F_{\mathrm{ext}}^{\mathrm{max}} & =\frac{M_{R}}{M_{R}+M_{H}} \sqrt{\frac{M_{R}+M_{H}}{M_{R} M_{H}}} \sqrt{K} \dot{x}_{R}^{0} M_{H} \\
& =\sqrt{\frac{M_{R} M_{H}}{M_{R}+M_{H}}} \sqrt{K} \dot{x}_{R}^{0} .
\end{aligned}
$$

For $M_{R} \gg M_{H}$ this reduces to ${ }^{17}$

$$
F_{\mathrm{ext}}^{\max }\left(M_{R} \gg M_{H}\right)=\sqrt{K M_{H}} \dot{x}_{R}^{0} .
$$

Equation (5) shows that for a robot with significantly larger reflected inertia than the human head, the only influencing variables consist of contact stiffness, the impact velocity, and the mass of the human head. The robot mass no longer plays a role in the contact force. The intuitive analogy given by Haddadin et al. (2007b) of "Being hit at a certain velocity by an infinitely large robot is basically the same as if the human is running at this particular velocity against a rigid wall" is therefore confirmed.

In order to be able to judge more generically the influence of the reflected inertia of a particular robot during an impact with a mass-spring complex, we introduce the inertial saturation coefficient

17. Assuming a simplified decoupling of the head from the torso, which holds for the short duration of the impact. For the post-impact phase, neck stiffness and body inertia must be considered, which complicates the analysis significantly.
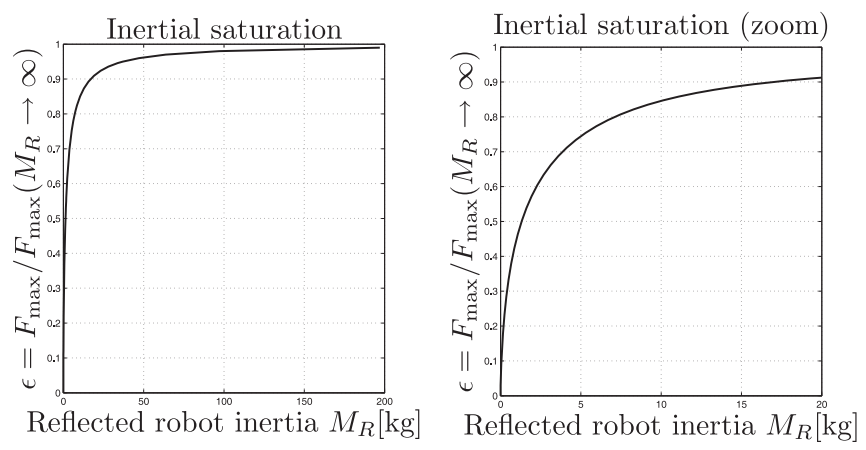

Fig. 11. The inertial saturation coefficient $\epsilon$ describes the effect that the robot mass has on the maximum contact force during an impact between a robot and a human. A reflected inertia of approximately $17 \mathrm{~kg}$ causes already $90 \%$ of the maximum possible contact force.

$$
\begin{aligned}
\epsilon & :=\frac{F_{\mathrm{ext}}^{\max }}{F_{\mathrm{ext}}^{\max }\left(M_{R} \gg M_{H}\right)} \\
& =\sqrt{\frac{M_{R}}{M_{R}+M_{H}}} \leq 1 .
\end{aligned}
$$

This quantity describes (independently of the contact stiffness and impact velocity) up to what percentage of the maximum (saturated) contact force is generated by a particular robot, cf. Figure 11. Therefore, it is possible to determine the maximum allowable force level (as a percentage of the saturation force), 

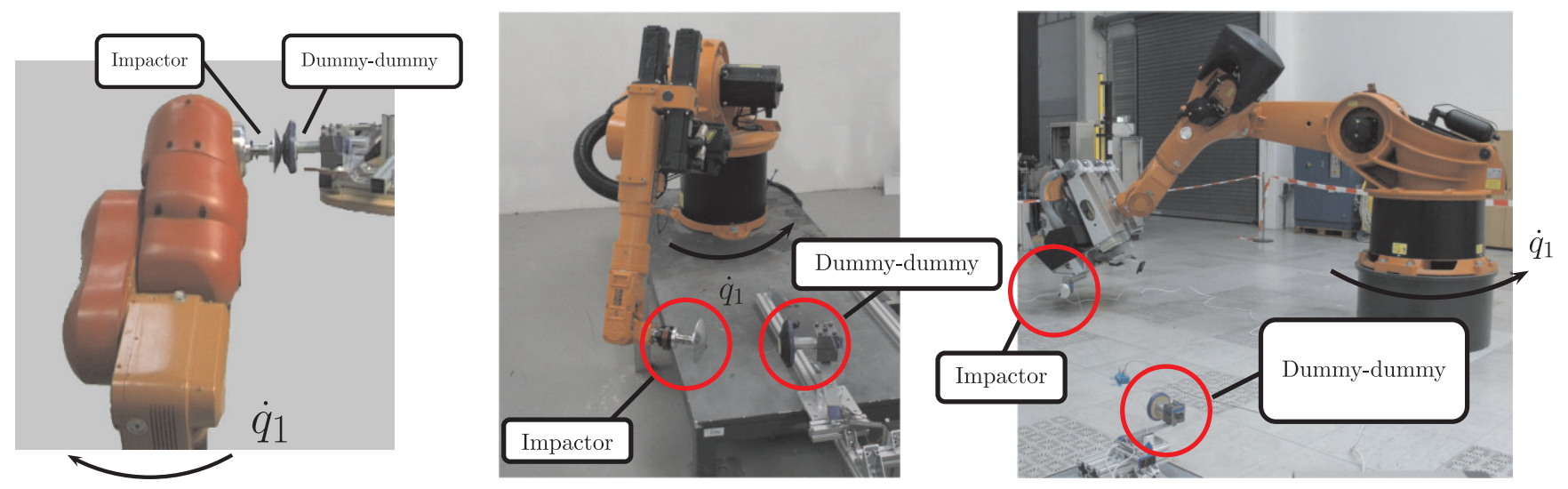

Fig. 12. Setup of the impact tests with the KUKA KR3-SI (left), KUKA KR6 (middle), and KUKA KR500 (right). Reflected inertias in the direction of impact were $\{12,67,1,870\} \mathrm{kg}$. The evaluation was based on a simple replication of a crash test dummy head which was designed such that it reproduces the same numerical HIC values and contact forces as a HIII (Haddadin et al. 2008b).

which may be used to define the requirements for the maximum reflected inertia of the robot.

\section{The Role of the Robot Mass and Velocity: Non-constrained Blunt Impacts}

In this section the experimental confirmation of the statements given in Section 5.6 regarding the saturation of HIC with robot mass is presented. Our results indicate that HIC and similar criteria which refer to very severe injury have low values. Therefore, it is crucial to evaluate lower severity injuries and find adequate measures for them. The evaluation of HIC and related criteria basically reduces the range of injury severities to be investigated drastically. A closer look should be taken at these lower range injuries.

As the recorded contact forces during all impact experiments were in the kilonewton range, we identified fractions of facial and cranial bones as a potential injury worth investigation due to their correlation to contact force.

\subsection{Evaluated Robots}

In order to cover a wide range of robots and to be able to verify the saturation effect explained in Section 5.6, we compared a $54 \mathrm{~kg}$ (small), $235 \mathrm{~kg}$ (medium), and a $2,350 \mathrm{~kg}$ (large) $\operatorname{robot}^{18}$ with the LWRIII, cf. Figure 12. The industrial robot tests were carried out with a simplified setup (denoted as Dummy-dummy), mimicking a HIII dummy head ${ }^{19}$.

18. These robots were the $54 \mathrm{~kg}$ KUKA KR3-SI, the $235 \mathrm{~kg}$ KUKA KR6, and the 2,350 kg KUKA KR500.

19. This was due to the high costs of crash tests at certified facilities. The validity of the setup is shown by Haddadin et al. (2008b). Currently, preparations are underway to confirm these statements with crash tests in cooperation with the ADAC, i.e. again with certified testing equipment.

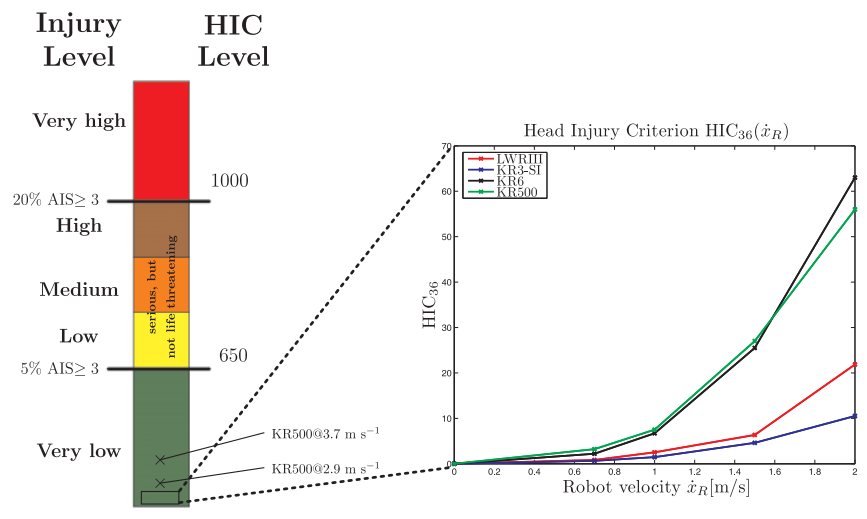

Fig. 13. Resulting $\mathrm{HIC}_{36}$ values at varying impact velocities for all robots, rated according to the EuroNCAP Assessment Protocol and Biomechanical Limits.

A safety feature of the small robot is the safeguarding of the tool by means of an intermediate flange with breakaway function, triggering the emergency stop in case the contact force at the TCP exceeds a certain threshold ${ }^{20}$. In combination with the mounted impactor the weight of the flange-impactor complex is $1.4 \mathrm{~kg}$.

\subsection{HIC and Impact Forces}

In Figure 13 the resulting HIC values for the different robots are depicted and classified according to the EuroNCAP. The

20. The initiated emergency stop is a Category 0,1 stop according to DIN EN 60204. Category 0 stop means that the drives are immediately switched off and the brakes engage at the same time. A Category 1 stop lets the robot halt with a hard stop trajectory without using the brakes. 

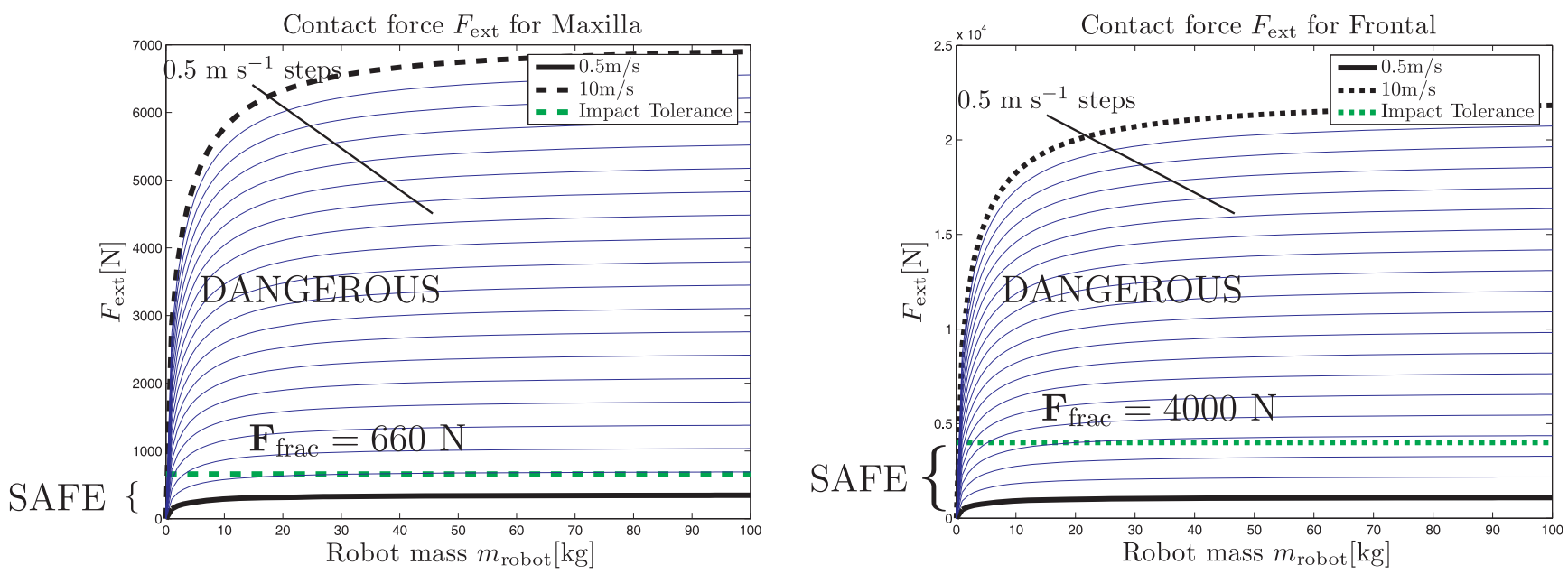

Fig. 14. Contact forces for simulated impacts between a robot and the frontal area (left) and the maxilla (right) showing the dependency on the robot mass and velocity. The impact velocity steps are $0.5 \mathrm{~m} \mathrm{~s}^{-1}$. Similar to HIC, a saturation effect can be observed such that for this conservative estimation, impact forces of $1 \mathrm{~m} \mathrm{~s}^{-1}$ can already potentially break the maxilla.

values for the small robot are even lower than for the LWRIII as the intermediate flange decouples the impactor from the entire robot at the moment of impact. Therefore, only the flangeimpactor complex is involved in the impact. The saturation effect explained in Section 5.6 is clearly observed, as the numerical values for the medium and large robot do not differ significantly. The simulation results presented in Figure 10 should be considered as conservative, since the actual saturation value is noticeably lower than predicted by simulation. This result indicates a very low potential injury. The probability of a resulting injury level of AIS $\geq 3$ according to NHTSA (1997) is maximally about $0.15 \%$. The HIC for the large robots measured at $80 \%$ and $100 \%$ of the maximum joint velocity, corresponding to a Cartesian velocity of 2.9 and $3.7 \mathrm{~m} \mathrm{~s}^{-1}$, was 135 and 246. This means that even an impact of such a large robot as the 2,350 $\mathrm{kg}$ robot cannot pose a significant threat to the human head by means of typical severity indices from automobile crash testing. The injury level for these values are located in the green area and the probability of AIS $\geq 3$ injuries are $1.2 \%$ and $3.6 \%$ for the faster impacts with the large robot, cf. Figure 13.

Our results clearly indicate that HIC and similar criteria are apparently not appropriate measures of possible injuries in robotics relevant to human-robot interaction ${ }^{21}$. This necessitates the investigation of other injury mechanisms of lower

21. In contrast to the requirements in human-robot interaction we claim that in competitive robotics a robot must not be more dangerous than a human (Haddadin et al. 2007c, 2009). In order to be a peer opponent such as in the ultimate goal of RoboCup, the robots require similar physical capabilities to a human, leading to extraordinary speed requirements. Since such impacts are approaching velocities at which automobile crash testing takes place, injury measures such as the HIC may be used to evaluate possibly occurring injury there. severity such as fracturing of facial and cranial bones, which could occur during human-robot collisions. This is indicated by recorded contact forces of the discussed impact tests which were within the fracture tolerance of these bones (Haddadin et al. 2008b,c).

In Figure 14 the dependencies of the impact force on the robot mass and velocity (the robot is assumed to move with constant velocity) for the frontal bone and the maxilla are visualized. Since the goal of this work is to establish safety limits to prevent bone fractures, the simulations were carried out for worst-case scenarios ${ }^{22}$. For all bones ${ }^{23}$, except the frontal bone, it appears that from the saturation mass value and above, a velocity between $0.5-1.0 \mathrm{~m} \mathrm{~s}^{-1}$ is sufficient to cause fractures. The frontal bone on the other hand is very fracture/injury resistant, able to withstand impacts approximately up to $2 \mathrm{~m} \mathrm{~s}^{-1}$. Furthermore, for robots with less than $5 \mathrm{~kg}$ reflected inertia at the moment of impact, the velocity can be significantly higher without exceeding the limit contact force. For weaker bones such as the maxilla, impact speeds of $2 \mathrm{~m} \mathrm{~s}^{-1}$ already pose a major threat for fracture even for low-inertia robots.

The experiments described in Table 1 validate our assumption of a conservative but nevertheless realistic upper bound. According to Kallieris (2007) the correlation between kinetic impact energy and injury severity by means of frontal fractures for cadaver head drop tests on ground were observed.

Below $50 \mathrm{~J}$ usually no fractures occur. An impact velocity of $2 \mathrm{~m} \mathrm{~s}^{-1}$ would yield a kinetic energy of $10 \mathrm{~J}$ at a drop height of $0.2 \mathrm{~m}$. The impact force would be $4.4 \mathrm{kN}$ for the assumed stiffness of the frontal bone in Figure 14 (left), im-

22. The contact stiffness is assumed to be the worst-case found in the literature. 23. Simulations for other facial and cranial bones were also carried out and show similar behavior. 
Table 1. Drop Tests with Cadaver Heads

\begin{tabular}{ll}
\hline Energy $(\mathrm{J})$ & Resulting injury \\
\hline $50-100$ & $\begin{array}{l}\text { Drop from 1-2 } \mathrm{m} \text { height }\left(4.6-9.6 \mathrm{~m} \mathrm{~s}^{-1}\right) . \\
\text { Results in a simple linear fracture of AIS }=2, \\
\text { or a more severe AIS }=3 \text { injury }\end{array}$ \\
$\begin{array}{l}\text { Complicated fracture with AIS } \geq 3 \\
\text { injury severity }\end{array}$ \\
$\begin{array}{l}\text { Vascular injury (leading to hematoma). } \\
\text { Combination of AIS for skull and } \\
\text { brain AIS }>3\end{array}$ \\
\hline
\end{tabular}

plying a fracture already at $10 \mathrm{~J}$. This can be explained by the conservative estimation of the frontal stiffness, which neglects the comparatively slowly increasing force in the beginning of an impact (Allsop et al. 1988; Allsop et al. 1991). Therefore, Figure 14 shows overestimation of the resulting injury. However, it is shown by Melvin (1980) that frontal fracture can already occur at 2-3 kN for smaller contact areas. Schneider and Nahum (1972) indicates that frontal fractures can already occur $\mathrm{at}^{24} 37 \mathrm{~J}$. Owing to the very significant biomechanical variation found in the literature we chose to assume the most conservative contact stiffness, leading to an upper bound which is conservative in the range of factor two. Compared with ISO10218, which is conservative in the range of more than an order of magnitude (for both the force and velocity), the suggested limits avoid the overly stringent limitation of robot performance demanded ${ }^{25}$ by ISO10218. The events which can happen after a fracture occurs are discussed by Haddadin et al. (2008b).

The next section contains clamping simulations based on measurements with several industrial robots to examine the large injury potential posed by environmental constraints.

\section{The Role of the Robot Mass and Velocity: Constrained Blunt Impacts}

Upon analyzing free impacts in detail, the influence of clamping is outlined for the head and chest. In Table 2 the clamping forces of the maxilla for impacts at $2 \mathrm{~m} \mathrm{~s}^{-1}$ for all robots ${ }^{26}$ in their particular impact configuration are listed. Each robot reacts to the collision by braking with maximum torque and continuing so until contact with the head is lost. The simulations show the vast influence of the robot mass. The small

24. An impactor was used, i.e. drop tests with a pre-defined impactor mass were carried out.

25 . Note that ISO10218 imposes a velocity limit of $0.25 \mathrm{~m} \mathrm{~s}^{-1}$, corresponding to a drop height of $2 \mathrm{~mm}$.

26. For this simulation the small robot is assumed to have no intermediate flange with breakaway function.
Table 2. Impact Forces with Clamping Obtained for the Maxilla: Category 0 Denotes Stopping With Brakes and Category 1 Denotes the Fastest Possible Stop Without Brakes

\begin{tabular}{|c|c|c|}
\hline Robot & Contact force & $\begin{array}{c}\text { Maxilla } \\
\text { fracture? }\end{array}$ \\
\hline LWRIII & $0.6 \mathrm{kN} @ 1 \mathrm{~m} \mathrm{~s}^{-1}$ & No \\
\hline LWRIII & $1.2 \mathrm{kN} @ 2 \mathrm{~m} \mathrm{~s}^{-1}$ & Yes \\
\hline KR3 & $2.2 \mathrm{kN} @ 2 \mathrm{~m} \mathrm{~s}^{-1}$ & Yes \\
\hline KR6 (Category 0\&1) & $5.1 \mathrm{kN} @ 2 \mathrm{~m} \mathrm{~s}^{-1}$ & Yes \\
\hline KR500 (Category 0\&1) & $23.6 \mathrm{kN} @ 2 \mathrm{~m} \mathrm{~s}^{-1}$ & Yes \\
\hline Robot & Contact force & $\begin{array}{c}\text { Frontal } \\
\text { fracture? }\end{array}$ \\
\hline LWRIII & $3.5 \mathrm{kN} @ 2 \mathrm{~m} \mathrm{~s}^{-1}$ & No \\
\hline KR3 & $6.9 \mathrm{kN} @ 2 \mathrm{~m} \mathrm{~s}^{-1}$ & Yes \\
\hline KR6 (Category 0\&1) & $16.3 \mathrm{kN} @ 2 \mathrm{~m} \mathrm{~s}^{-1}$ & Yes \\
\hline KR500 (Category 0\&1) & $86.3 \mathrm{kN} @ 2 \mathrm{~m} \mathrm{~s}^{-1}$ & Yes \\
\hline
\end{tabular}

robot produces almost twice the contact force that the LWRIII generate ${ }^{27}$. However, all robots, even the low-inertia LWRIII, can potentially break the maxilla at $2 \mathrm{~m} \mathrm{~s}^{-1}$. In addition, the applied model is no longer valid after the fracture occurs, as the resistance on the robot is dramatically lowered, possibly causing even more (under certain circumstances even fatal) severe secondary injury. However, for the LWRIII a safe velocity of at least $1 \mathrm{~m} \mathrm{~s}^{-1}$ is possible.

In Table 3 the resulting compression criterion (CC), the viscous criterion (VC), and the clamping forces are listed for impacts at $2 \mathrm{~m} \mathrm{~s}^{-1}$ with a clamped chest. CC is the deflection of the chest and $\mathrm{VC}$ the relative deflection multiplied by intrusion velocity, see Haddadin et al. (2007b). The EuroNCAP injury level (Haddadin et al. 2007b) is indicated for the CC and VC with corresponding colors. For the CC the AIS level is additionally obtained by another available mapping ${ }^{28}$ and is denoted in brackets. The injury level of the $\mathrm{CC}$ clearly shows that increasing robot mass leads to a higher probability of injury level. Similar conclusions can be drawn from the contact force and its correlating injury level, showing that both criteria are sensitive predictors of injury for the chest in the case of clamping. The $\mathrm{VC}$, in contrast, is due to the low velocities only relevant for the large robot, as even with low intrusion velocity the deflection dominates the VC value in this case. Similar to the head we can see that the chest is exposed to an increasing threat with growing robot mass if the human is clamped. More detailed simulations showing specifically the correlation

27. The relationship between motor torque and inertia scales is disadvantageous with increasing dimensions.

28. For details refer to Haddadin et al. (2007b, 2008c). 
Table 3. Simulated values for chest severity indices and corresponding AIS values obtained for the human chest (right). Category 0 denotes stopping with brakes and Category 1 denotes the fastest possible stop without brakes.

\begin{tabular}{llll}
\hline Robot & $\mathrm{CC}(\mathrm{mm})$ & $\mathrm{VC}\left(\mathrm{m} \mathrm{s}^{-1}\right)$ & $F_{\text {ext }}^{x}[\mathrm{~N}]$ \\
\hline LWRIII & $14.4(0.0)$ & 0.035 & $741.6(1.3)$ \\
KR3 (Category 0) & $31.2(0.0)$ & 0.1 & $851.9(1.4)$ \\
KR6 (Category 0) & $65.5(2.0)$ & 0.25 & $2,836.1(2.7)$ \\
KR6 (Category 1) & $66.6(2.1)$ & 0.25 & $2,904.6(2.7)$ \\
KR500 (Category 0) & $228.0(6.0)$ & 0.84 & $14,282.0(6.0)$ \\
KR500 (Category 1) & $245.0(6.0)$ & 0.89 & $15,491.0(6.0)$ \\
\hline
\end{tabular}

between impact speed and injury criterion for each robot are also outlined by Haddadin et al. (2008c).

After this investigation of dynamic blunt impacts with and without clamping, the problem of quasi-static loading will be discussed as a case study on the LWRIII. The resulting methodology of investigation is applicable to any robot.

\section{Singularity Forces During Quasistatic Constrained Impact}

For impact configurations with large levers, robots of similar inertias (and maximum joint torques) to the LWRIII do not pose a potential threat with regards to HIC (Haddadin et al. 2007b). However, the nearly fully outstretched arm poses a significant injury threat which shall be evaluated more in detail, cf. Figure 15.

The maximum nominal torques for a given robot are represented by a hyper-rectangle. The corners of this hyperrectangle are then transformed via the pseudo-inverse of the transposed Jacobian to the corners of a hyper-polygon of Cartesian forces. In order to acquire the maximal applicable force in the relevant worst-case direction, the corresponding hyper-rectangle corner has to be evaluated. Here, we use the collision detection (CD) mentioned in Section 5.4 and described in detail by De Luca et al. (2006) and Haddadin et al. (2008a). Its detection threshold $\boldsymbol{\tau}_{\text {det }}$ for the external joint torque of the robot is defined as a percentage of the maximum nominal joint torque $\boldsymbol{\tau}_{\max }$ (e.g. 2\%) which allows us to obtain the detection threshold of the contact force:

$$
\begin{aligned}
\boldsymbol{\tau}_{\mathrm{det}} & =0.02 \boldsymbol{\tau}_{\max } \\
\rightarrow \mathbf{F}_{\mathrm{det}} & =0.02 \mathbf{F}_{\max }=\mathbf{J}^{\mathrm{T} \#} \boldsymbol{\tau}_{\mathrm{det}},
\end{aligned}
$$

where $\mathbf{J}^{\mathrm{T} \#}$ is the pseudo-inverse of the transposed manipulator Jacobian ${ }^{29}$. Theoretically, of the configuration boundaries

29. Note, that since the torque $\boldsymbol{\tau}_{\text {det }}$ is produced only by a TCP force, any generalized pseudo-inverse will lead to the same value of $\mathbf{F}_{\text {det }}$.

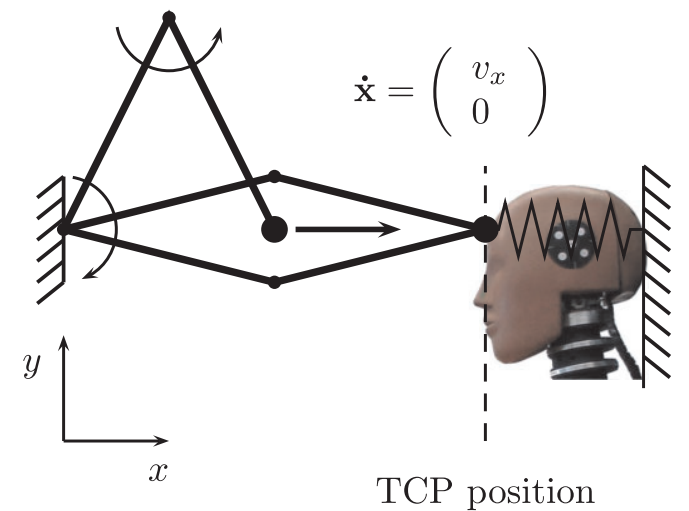

Fig. 15. Impact configuration for LWRIII dummy crash tests. Clamping the human with the robot in near-singular (almost outstretched) configuration. This is due to reconfiguration from "elbow up" to "elbow down" or vice versa.

which can cause fractions of facial and cranial bones, the reconfiguration from "elbow up" to "elbow down" is the most dangerous case. The robot can be commanded in such a way that it passes the fully outstretched position if the clamped head is contacted close to the singularity. Since the human head would be clamped only very slowly due to the low Cartesian velocities close to the singularity, an acceleration-based criterion such as the HIC cannot indicate the force that is exerted on the head. Therefore, such criteria drop out entirely for this analysis and we use contact forces and related bone fractures as injury indicators ${ }^{30}$.

In Figure 16 the maximal force which can be exerted on a human maxilla by a rigid, slowly moving robot (no dynamic

30. This statement cannot be made for high-speed constrained impacts at the current state since the human head is not a rigid body and it cannot be excluded that an acceleration occurs during such impact. We are currently preparing impact tests to be able to formulate statements concerning this issue. 

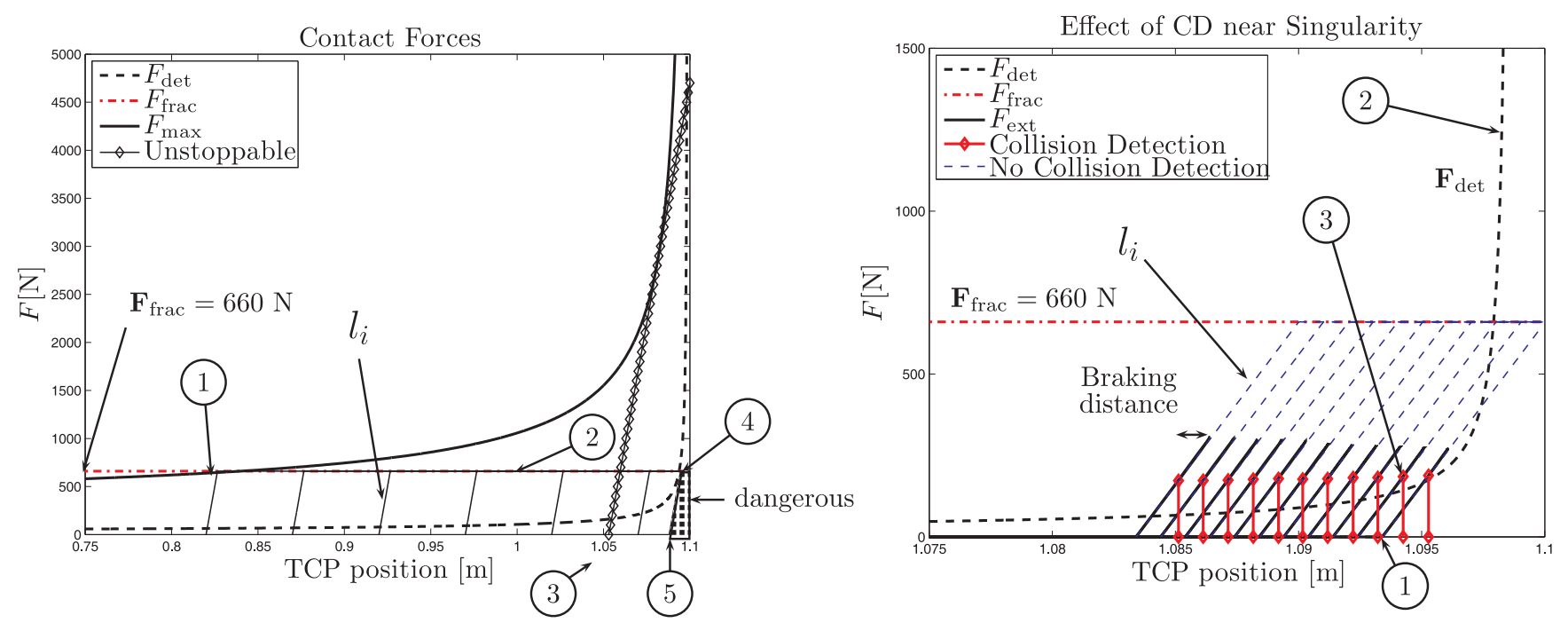

Fig. 16. Clamping of the human head with a rigid manipulator. The chosen bone for this analysis is the maxilla, whereas the theoretical analysis for the reconfiguration of the manipulator is also shown (left). For better illustration the collision threshold is set to $10 \%$ in this plot. The evaluation of the collision detection (CD) schemes with a full dynamic simulation of the LWRIII confirms their benefit (right). This reconfiguration trajectory (cf. Figure 15) was carried out at maximum joint velocity of the LWRIII at $120^{\circ} \mathrm{s}^{-1}$. The deviation of the behavior of the LWRIII from the rigid case is mainly due to the intrinsically flexible joints and the contact modeling. In the right plot the collision threshold is set to the currently lowest achievable value of $2 \%$.

forces) is analyzed. The stiffness of the maxilla ${ }^{31}$ is of the order of $10^{5} \mathrm{~N} \mathrm{~m}^{-1}$ according to Nyquist et al. (1986), Allsop et al. (1988) and McElhaney et al. (1972). Thus, the force will linearly increase with position after contact, as represented in Figure 16 for several collision points along the lines $l_{i}$. The linear forces are displayed only up to the limit at which the bone will break (2), denoted by $\mathbf{F}_{\text {frac }}=660 \mathrm{~N}$. The curve $\mathbf{F}_{\max }$ represents the maximal force that can be exerted by the robot, which goes towards infinity when approaching the singularity. If this curve is above $\mathbf{F}_{\text {frac }}$ and if $\mathbf{F}_{\text {frac }}$ is exceeded before reaching the singularity for a given collision point (depending on the slope of $l_{i}$ ), the bone will break. For the considered case, this would happen starting with (1), i.e. more than $27 \mathrm{~cm}$ before reaching the singularity. Starting with (3), there does not exist a hypothetical equilibrium point, meaning that the considered stiffness cannot stop the robot from reaching the singularity. Using the collision detection with a threshold of $0.1 \tau_{\max }$, the maximal forces are lowered, as displayed by the curve $\mathbf{F}_{\text {det }}$. In this case, the critical region is substantially reduced to about $2 \mathrm{~cm}$ before the singularity (4). Restricting the workspace of the arm such that this configuration is not reached, poses no significant limitation to usual applications. The limit safe configuration ${ }^{32}$ is denoted by (5). This analysis

31. As the variation of data obtained by human cadaver tests is quite large and this data cannot be applied to children or elderly people, it has to be treated carefully.

32. With an ideal collision detection and an infinitely fast stopping robot. is carried out with all facial and cranial bones listed in Haddadin et al. (2008b) and yields similar observations for each of them.

After this rigid-robot evaluation, the full-dimensional simulation, especially including its intrinsic joint compliance for the LWRIII at maximum joint velocity, is given and discussed.

For the results shown in Figure 16 (right) we assumed a feasible collision detection threshold of $0.02 \tau_{\max }$. The implemented collision reaction strategy immediately sets the desired position to a resting position $50 \mathrm{~mm}$ before reaching the singularity. In contrast to the rigid robot there exists no significant workspace restriction, since even the last possible impact location (1) that could theoretically lead to the fracture force $\mathbf{F}_{\text {frac }}$ can be handled by the collision detection. The theoretical rigidcase collision threshold (2) close to the singularity is slightly below that obtained from the complete dynamic simulation (3) presumably due to the elasticity in the joints of the real robot. Furthermore, the Cartesian braking distance decreases as the contact point approaches the singularity. This is due to the duality of Cartesian velocity and force. After the collision detection activation and the subsequent braking distance, the robot switches its Cartesian velocity direction and comes to a rest position $50 \mathrm{~mm}$ before the singularity. For clarity Figure 17 denotes the time course for such a constrained impact. It shows how the collision detection and reaction can limit the contact force to subcritical values. In this particular simulation the human is standing $16 \mathrm{~mm}$ before the singularity with enough space remaining to achieve the necessary fracture force. 


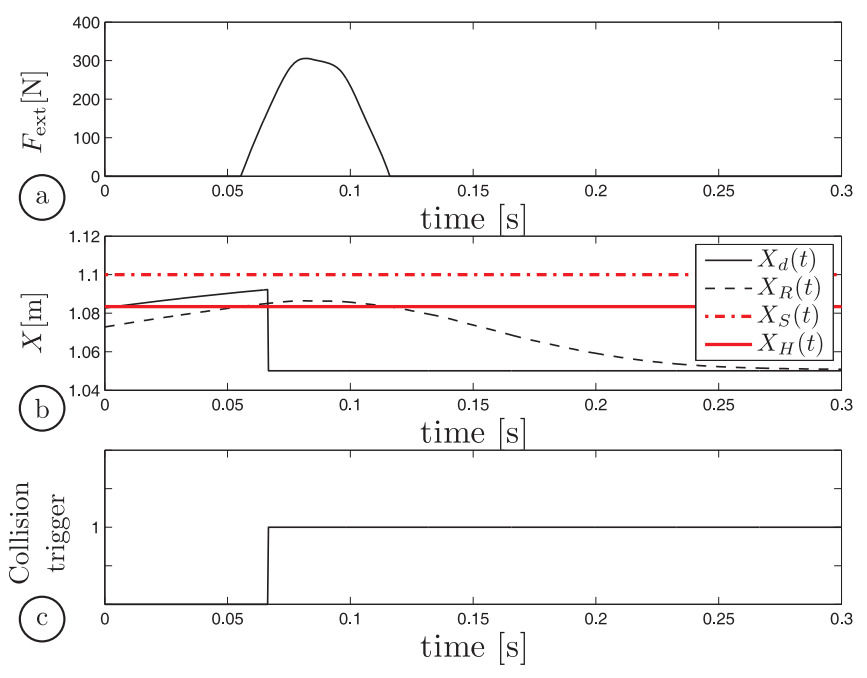

Fig. 17. Time courses for the constrained impact with the full dynamic model of the LWRIII and the human head close to the singularity. The contact stiffness corresponds to the maxilla at $10^{5} \mathrm{~N} \mathrm{~m}^{-1}$. (a) The time course of the contact force, pointing out that the collision detection prevents an exceedance of the threshold force of $660 \mathrm{~N}$. (b) The desired trajectory $X_{D}(t)$, the robot position $X_{R}(t)$, the position of the singularity $X_{S}(t)$, and the position of the human $X_{H}(t)$. (c) The collision detection signal which triggers the reset of $X_{D}(t)$ in (b).

In a well-structured environment, such as the robot workcell, this type of singularity clamping can indeed be avoided. However, robots such as the LWRIII are supposed to act as an industrial robotic co-worker, continuously working together with humans and sharing the ever-changing workspace. Singularity clamping situations due to human error can therefore not be excluded.

\section{Possible Injuries: A Summary}

Up to now only isolated issues and mechanisms of robot safety were discussed and introduced in the robotics literature. In order to have an overview of the potential injury threats depending on the current state of the robot and the human, a classification of these mechanisms, governing factors of the particular process, and possible injuries are proposed in Figure 18. Physical contact can be divided into two fundamental subclasses: quasi-static and dynamic loading ${ }^{33}$. Fundamental differences in injury severity and mechanisms are also observed if a human is (partially) constrained or not. For the quasi-static case we differentiate between near-singular and non-singular

33. We consider only injuries for typical robot velocities and no hypothetic extreme cases. As pointed out in previous sections and Haddadin et al. (2007b, 2008 b), injury potential vastly increases with the impact velocity of the robot. clamping as already outlined. The last differentiation separates injuries caused by blunt contact from those induced by tools or sharp surface elements.

Each class of injury is characterized by possible injuries (PI), worst-case factors (WCF) and their worst-case range (WCR). WCF are the main contributors to the worst-case, such as maximum joint torque, the distance to singularity or the robot speed. The worst-case range indicates the maximum possible injury depending on the worst-case factors. In addition to the classification of injury mechanisms for each such class, suggestions for injury measures (IM) are also given. They are specific injury measures which appear appropriate, useful, and applicable for the classification and measurement of injury potentially occurring during physical human-robot interaction.

For example, (1) represents blunt clamping in the nearsingular configuration, cf. Figure 18. As already shown, even for low-inertia robots this situation could become dangerous and is therefore a possible serious threat with almost any robot on a fixed base within a (partially) confined workspace. Possible injuries are fractures and secondary injuries, e.g. caused by penetrating bone structures or an injured neck if the trunk is clamped but the head is free. This would mean that the robot pushes the head further while the trunk remains in its position. Another possible threat is shearing off a locally clamped human along an edge. Appropriate indices are the contact force and the compression criterion. (3) is the clamped blunt impact in a non-singular configuration. The injury potential is defined by the maximum joint torque $\boldsymbol{\tau}_{\max }$ and can range from no injury (as shown for the LWRIII) to severe injury or even death for high-inertia (and joint torque) robots. The robot stiffness does not contribute to the worst-case since a robot without collision detection would simply increase the motor torque to follow the desired trajectory. Therefore, robot stiffness only contributes to the detection mechanism by increasing the detection time. Furthermore, the contact force and the compression criterion are well suited to predict occurring injury. (8) denotes the classical free impact which was the first injury mechanism investigated in the robotics literature. This process is governed by the impact velocity and by the robot mass up to a saturation value. As shown by Haddadin et al. (2007b) and in Sections 5 and 6 a robot of any arbitrary mass cannot severely hurt a human head by means of impact related criteria from the automobile industry such as HIC. However, fractures, e.g. of facial bones, are likely to occur, but not all can be classified as a serious injury. Laceration by means of crushes and gashes are certainly worthwhile to be evaluated, especially with respect to service robotics. The contact force and the compression criterion are well suited severity criteria for this class. In order to evaluate lacerations, the energy density has to be considered.

The preceding evaluation of injuries is intended as a worstcase analysis for the described contact cases. The logical next step is to determine the appropriate countermeasures to be taken against each particular threat. (1)-(5) can be handled by a collision detection and reaction as described by De Luca 


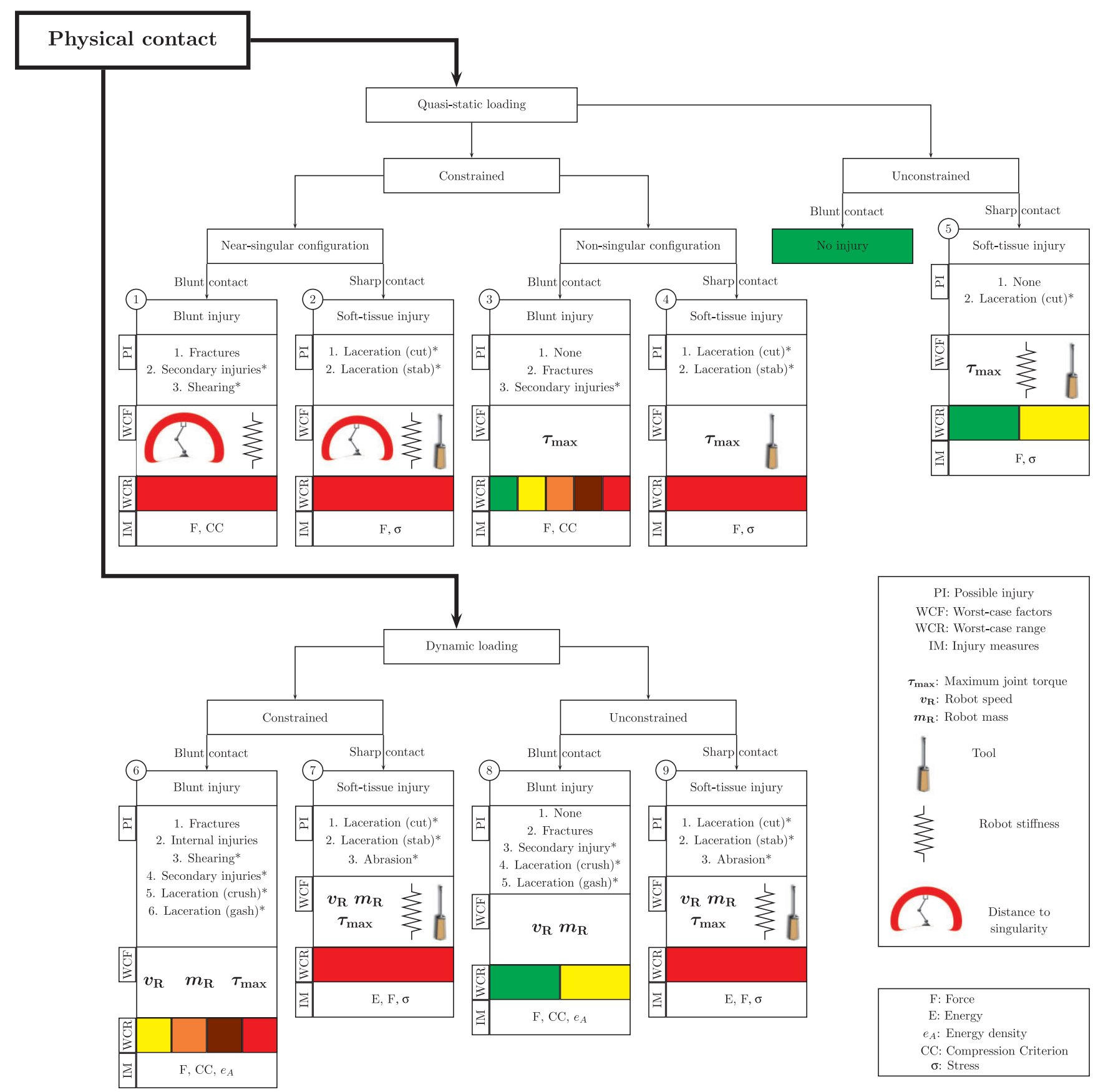

Fig. 18. Safety Tree showing possible injury (PI), major worst-case factors (WCF) and the possible worst-case range (WCR). A * indicates still ongoing topics of research. In addition a selection of relevant injury criteria are given for the head, chest, and soft-tissue injuries.

et al. (2006), Haddadin (2005), and Haddadin et al. (2008a). Good countermeasures in the case of (6) appear to be soft covering, lightweight design, and a fast and effective collision detection and reaction. (7) seems to be the most dangerous scenario one can think of and requires very special treatment. Safe robot speed, which gives the human enough time to react accordingly, is indispensable. Second, an effective collision detection and a safe and carefully selected collision reaction have to be embedded. Similar countermeasures are appropriate for (9). 


\section{Conclusion}

In this paper we have presented the first systematic evaluation and classification of possible injuries during physical humanrobot interaction. We have shown experimentally that potential injury of the head, occurring during a free blunt impact, would saturate with increasing robot mass. Above a certain mass, potential injury would only depend on the impact velocity. Thus, typical severity indices focusing just on the moment of impact such as the HIC are not appropriate measures of injury severity in human-robot interaction as usually a robot does not exceed these safety critical thresholds. This is due to the usually significantly lower velocities of the robots compared with velocities during impact tests carried out in automobile crash testing. In general, blunt head impacts without clamping at typical robot speeds up to $\approx 2 \mathrm{~m} \mathrm{~s}^{-1}$ are very unlikely to be life-threatening regardless of the weight of the robot. Chest impacts of the same kind are even less dangerous, as shown by real impact data. To the best of the authors' knowledge, they represent the first systematic experimental evaluation of possible injuries during robot-human impacts using standardized testing facilities. However, for the head, other serious injuries, such as fractures of facial and cranial bones, can (under conservative assumptions) occur already at moderate velocities and seem to be a more relevant injury mechanism. The appropriate injury indicator for this class of injury is not related to head acceleration but to impact forces.

Drastically different observations can be made in the case of clamping which was evaluated with respect to robot mass and impact velocity. In the case of clamping both the head and chest can be severely injured even leading to fatality for a large robot mass. Nevertheless, the low inertial properties of the LWRIII allow an impact velocity of up to $1 \mathrm{~m} \mathrm{~s}^{-1}$ without leading to any of the investigated injuries.

Apart from the discussed dynamic impacts, we have also shown that even low-inertia robots can become very dangerous in near-singular configurations in the case of a constrained impact. On the other hand we demonstrated the effectiveness of a collision detection and reaction scheme which can handle this threat.

Finally, we have classified relevant injury mechanisms, important factors governing each injury process and the worstcase injury level derived from it. This classification should be considered as a basis for further investigations, as well as a roadmap pointing out open issues and the variety of possible injury mechanisms in physical human-robot interaction.

Comparing the thresholds defined in ISO10218 with our measurements, it is clear that its definition is not based on biomechanical analysis. Such an evaluation leads to much higher tolerance values for blunt impacts. The intention of ISO10218 is to keep the velocity of the robot low in order to enable active avoidance of unintended contact with a human. If this is not possible, only very low exerted forces and power could avoid any kind of risk, i.e. ISO10218 defines truly conserva- tive safety requirements. However, this seems to be an overly stringent restriction of robot performance, while at the same time there is still a lack of a well-differentiated standard. Particular tools and their corresponding injuries which would demand even lower thresholds than currently required are not discussed in this standard. We recommend to define a more sophisticated and differentiated basis to achieve an optimal safety-performance tradeoff.

\section{Acknowledgment}

This work has been partially funded by the European Commission's Sixth Framework Programme as part of the projects SMERobot ${ }^{\mathrm{TM}}$ under grant no. 011838, PHRIENDS under grant no. 045359, and VIACTORS under grant no. 231554. Our special thanks go to Professor Dimitrios Kallieris for his valuable advice in the field of biomechanics and forensics. Furthermore, we would like to thank Professor Alessandro De Luca, Mirko Frommberger, Michael Strohmayr, and Oliver Eiberger for their great help and support. Last but not least we greatly appreciate the invaluable suggestions for improvement contributed by Dr Michael Suppa.

\section{Appendix: Index to Multimedia Extensions}

The multimedia extension page is found at http://www.ijrr.org

\section{Table of Multimedia Extensions}

\begin{tabular}{lll}
\hline Extension & Type & Description \\
\hline 1 & Video & $\begin{array}{l}\text { Crash test experiments with the } \\
\text { LWRIII at the German Automobile } \\
\text { Club ADAC. }\end{array}$ \\
2 & Video & $\begin{array}{l}\text { Human self experiments with the } \\
\text { LWRIII. } \\
3\end{array}$ \\
Video & $\begin{array}{l}\text { Crash test experiments with industrial } \\
\text { robots. }\end{array}$ \\
5 & Video & $\begin{array}{l}\text { Simulation of a constrained impact } \\
\text { close to a singularity. } \\
5\end{array}$ \\
& Video & $\begin{array}{l}\text { Soft-tissue experiments with the } \\
\text { LWRIII. }\end{array}$ \\
\hline
\end{tabular}

\section{References}

AAAM (1980). The Abbreviated Injury Scale. Barrington, IL, Association for the Advancement of Automotive Medicine. AAAM (1998). The Abbreviated Injury Scale (1990) Revision Update 1998. Barrington, IL, Association for the Advancement of Automotive Medicine. 
Albu-Schäffer, A., Haddadin, S., Ott, C., Stemmer, A., Wimböck, T. and Hirzinger, G. (2007a). The DLR Lightweight Robot@ lightweight design and soft robotics control concepts for robots in human environments. Industrial Robot Journal, 34: 376-385.

Albu-Schäffer, A., Ott, C. and Hirzinger, G. (2007b). A unified passivity-based control framework for position, torque and impedance control of flexible joint robots. The International Journal of Robotics Research, 26: 23-39.

Allsop, D. L., Perl, T. R. and Warner, C. Y. (1991). Force/ deflection and fracture characteristics of the temporoparietal region of the human head. Proceedings of the Stapp Car Crash Conference, November 1991, SAE Paper No. 912907, pp. 2009-2018.

Allsop, D. L., Warner, C. Y., Wille, M. G., Schneider, D. C. and Nahum, A. M. (1988). Facial impact response-a comparison of the Hybrid III dummy and human cadaver. Proceedings of the 32th Stapp Car Crash Conference, SAE Paper No. 881719, pp. 781-797.

Asimov, I. (1954). The Caves of Steel, A Robot Novel.

Bicchi, A., Bavaro, M., Boccadamo, G., De Carli, D., Filippini, R., Grioli, G., Piccigallo, M., Rosi, A., Schiavi, R., Sen, S. and Tonietti, G. (2008). Physical human-robot interaction: dependability, safety, and performance. International Workshop on Advanced Motion Control, pp. 9-14.

Bicchi, A. and Tonietti, G. (2004). Fast and soft arm tactics: dealing with the safety-performance trade-off in robot arms design and control. IEEE Robotics and Automation Magazine, 11: 22-33.

Corke, P. I. (1999). Safety of advanced robots in human environments. A discussion paper. Proceedings of IARP.

De Luca, A., Albu-Schäffer, A., Haddadin, S. and Hirzinger, G. (2006). Collision detection and safe reaction with the DLR-III lightweight manipulator arm. IEEE/RSJ International Conference on Intelligent Robots and Systems (IROS2006), pp. 1623-1630.

EuroNCAP (2004). European Protocol New Assessment Programme-Frontal Impact Testing Protocol.

Haddadin, S. (2005). Evaluation Criteria and Control Structures for safe Human-Robot Interaction. Master's thesis, Technical University of Munich \& German Aerospace Center (DLR).

Haddadin, S., Albu-Schäffer, A., De Luca, A. and Hirzinger, G. (2008a). Collision detection \& reaction: a contribution to safe physical human-robot interaction. IEEE/RSJ International Conference on Intelligent Robots and Systems (IROS2008), Nice, France.

Haddadin, S., Albu-Schäffer, A. and Hirzinger, G. (2007a). Approaching Asimov's 1st law. HRI Caught on Film, Proceedings of the 2nd ACM/IEEE International Conference on Human-Robot Interaction, Washington, DC, pp. 177184.

Haddadin, S., Albu-Schäffer, A. and Hirzinger, G. (2007b). Safety evaluation of physical human-robot interaction via crash-testing. Robotics: Science and Systems Conference (RSS2007).

Haddadin, S., Albu-Schäffer, A. and Hirzinger, G. (2008b). The role of the robot mass and velocity in physical humanrobot interaction-part I: unconstrained blunt impacts. IEEE International Conference on Robotics and Automation (ICRA2008), Pasadena, CA, pp. 1331-1338.

Haddadin, S., Albu-Schäffer, A. and Hirzinger, G. (2008c). The role of the robot mass and velocity in physical humanrobot interaction-part II: constrained blunt impacts. IEEE International Conference on Robotics and Automation (ICRA2008), Pasadena, CA, pp. 1339-1345.

Haddadin, S., Laue, T., Frese, U. and Hirzinger, G. (2007c). Foul 2050: thoughts on physical interaction in human-robot soccer. IEEE/RSJ International Conference on Intelligent Robots and Systems (IROS2007), San Diego, CA, pp. 32433250 .

Haddadin, S., Laue, T., Frese, U., Wolf, S., Albu-Schäffer, A. and Hirzinger, G. (2009). Kick it like a safe robot: requirements for 2050. Proceedings of Robotics and Autonomous Systems.

Heinzmann, J. and Zelinsky, A. (2003). Quantitative safety guarantees for physical human-robot interaction. The International Journal of Robotics Research, 22(7-8): 479504.

Hirzinger, G., Sporer, N., Schedl, M., Butterfaß, J. and Grebenstein, M. (2004). Torque-controlled lightweight arms and articulated hands: do we reach technological limits now? The International Journal of Robotics Research, 23(4-5): 331-340.

Ikuta, K., Ishii, H. and Nokata, M. (2003). Safety evaluation method of design and control for human-care robots. The International Journal of Robotics Research, 22(5): 281298.

ISO (2006). Robots for industrial environments - Safety requirements - Part 1: Robot. Standard ISO10218, International Organization for Standardization.

Kallieris, D. (2007). Personal communication.

Kulic, D. and Croft, E. (2007). Pre-collision strategies for human robot interaction. Autonomous Robots, 22(2): 149164.

Lim, H.-O. and Tanie, K. (2000). Human safety mechanisms of human-friendly robots: passive viscoelastic trunk and passively movable base. The International Journal of Robotics Research, 19(4): 307-335.

McElhaney, J., Stalnaker, R. and Roberts, V. (1972). Biomechanical aspects of head injury. Human Impact ResponseMeasurement and Simulation.

Melvin, J. (1980). Human tolerance to impact conditions as related to motor vehicle design. SAE Paper J885, April 1980.

Morita, T., Iwata, H. and Sugano, S. (1999). Development of human symbiotic robot: WENDY. IEEE International Conference on Robotics and Automation (ICRA1999), Detroit, MI, pp. 3183-3188. 
NHTSA (1997). Actions to reduce the adverse effects of air bags. FMVSS No. 208.

Nyquist, G. W., Cavanaugh, J. M., Goldberg, S. J. and King, A. I. (1986). Facial impact tolerance and response. Proceedings of the 30th Stapp Car Crash Conference, SAE Paper No. 861896, pp. 733-754.

Oberer, S. and Schraft, R. D. (2007). Robot-dummy crash tests for robot safety assessment. IEEE International Conference on Robotics and Automation (ICRA2007), Rome, Italy, pp. 2934-2939.

Schneider, D. C. and Nahum, A. M. (1972). Impact studies of facial bones and skull. Proceedings of the 16th Stapp Car Crash Conference, SAE Paper No. 720965, pp. 186-204.

Shin, D., Sardellitti, I. and Khatib, O. (2008). Hybrid actuation approach for human-friendly robot design. IEEE International Conference on Robotics and Automation (ICRA 2008), Pasadena, CA, pp. 1741-1746.

SMErobot (2009). Final report on proposal for new safety standards for collaborative robots. The European Robot Initiative for Strengthening the Competitiveness of SMEs in Manifacturing.
United Auto Workers (2004). Review of robot injuries-one of the best kept secrets. National Robot Safety Conference, Ypsilanti, MI.

Vanderborght, B., Verrelst, B., Ham, R. V., Damme, M. V., Lefeber, D., Duran, B. and Beyl, P. (2006). Exploiting natural dynamics to reduce energy consumption by controlling the compliance of soft actuators. The International Journal Robotics Research, 25(4): 343-358.

Versace, J. (1971). A review of the severity index. Proceedings of the 15th Stapp Conference, SAE Paper No. 710881, pp. 771-796.

Wolf, S. and Hirzinger, G. (2008). A new variable stiffness design: matching requirements of the next robot generation. IEEE International Conference on Robotics and Automation (ICRA 2008), Pasadena, CA, pp. 1741-1746.

Yamada, Y., Hirasawa, Y., Huand, S. and Umetani, Y. (1996). Fail-safe human/robot contact in the safety space. IEEE International Workshop on Robot and Human Communication, pp. 59-64.

Zinn, M., Khatib, O. and Roth, B. (2004). A new actuation approach for human friendly robot design. The International Journal of Robotics Research, 23: 379-398. 